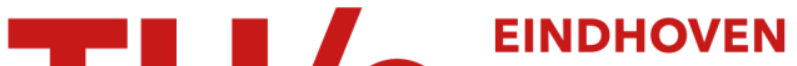 UNIVERSITY OF TECHNOLOGY
}

\section{Solving sparse polynomial optimization problems with chordal structure using the sparse, bounded-degree sum-of-squares hierarchy}

Citation for published version (APA):

Marandi, A., de Klerk, E., \& Dahl, J. (2020). Solving sparse polynomial optimization problems with chordal structure using the sparse, bounded-degree sum-of-squares hierarchy. Discrete Applied Mathematics, 275, 95110. https://doi.org/10.1016/j.dam.2017.12.015

DOI:

10.1016/j.dam.2017.12.015

Document status and date:

Published: 31/03/2020

\section{Document Version:}

Accepted manuscript including changes made at the peer-review stage

\section{Please check the document version of this publication:}

- A submitted manuscript is the version of the article upon submission and before peer-review. There can be important differences between the submitted version and the official published version of record. People interested in the research are advised to contact the author for the final version of the publication, or visit the DOI to the publisher's website.

- The final author version and the galley proof are versions of the publication after peer review.

- The final published version features the final layout of the paper including the volume, issue and page numbers.

Link to publication

\footnotetext{
General rights

- You may freely distribute the URL identifying the publication in the public portal. follow below link for the End User Agreement:

www.tue.nl/taverne

Take down policy

If you believe that this document breaches copyright please contact us at:

openaccess@tue.nl

providing details and we will investigate your claim.
}

Copyright and moral rights for the publications made accessible in the public portal are retained by the authors and/or other copyright owners and it is a condition of accessing publications that users recognise and abide by the legal requirements associated with these rights.

- Users may download and print one copy of any publication from the public portal for the purpose of private study or research.

- You may not further distribute the material or use it for any profit-making activity or commercial gain

If the publication is distributed under the terms of Article $25 \mathrm{fa}$ of the Dutch Copyright Act, indicated by the "Taverne" license above, please 


\title{
Solving sparse polynomial optimization problems with chordal structure using the sparse bounded-degree sum-of-squares hierarchy
}

\author{
Ahmadreza Marandi ${ }^{\mathrm{a}}$, Etienne de Klerk ${ }^{\mathrm{b}, \mathrm{c}}$, Joachim Dahl ${ }^{\mathrm{d}}$ \\ ${ }^{a}$ Department of Industrial Engineering and Innovation Sciences, Eindhoven University of Technology, The \\ Netherlands \\ ${ }^{b}$ Tilburg School of Economics and Management, Tilburg University, The Netherlands \\ ${ }^{c}$ Delft Institute of Applied Mathematics, Delft University of Technology, The Netherlands \\ ${ }^{d}$ MOSEK ApS, Copenhagen O, Denmark
}

\begin{abstract}
The sparse bounded degree sum-of-squares (sparse-BSOS) hierarchy of Weisser, Lasserre and Toh [Math Prog Compt:1-32, to appear] constructs a sequence of lower bounds for a sparse polynomial optimization problem. Under some assumptions, it is proved by the authors that the sequence converges to the optimal value. In this paper, we modify the hierarchy to deal with problems containing equality constraints directly, without eliminating or replacing them by two inequalities. We also evaluate the sparse-BSOS hierarchy on a well-known bilinear programming problem, called the pooling problem, as well as a discrete-time optimal control problem.
\end{abstract}

Keywords: Polynomial optimization, Sparse sum-of-squares hierarchy, Semi-definite programming, Pooling problem, Chordal sparsity structure, Discrete-time optimal control

2010 MSC: 90C20, 90C22, 90C26, 90C30

\section{Introduction}

A polynomial optimization problem (POP) is a mathematical optimization problem in which all constraints and the objective function are multi-variate polynomials. POPs include non-convex quadratic programming problems, which were proved to be $N P$-hard by Pardalos and Vavasis [26].

Many approaches are available for constructing lower bounds for the optimal value of a POP (denoted by $f^{*}$ ). Kim, Kojima and Waki 18 proposed a relaxation of a POP using a generalized

Email addresses: a.marandi@tue.nl (Ahmadreza Marandi), e.deklerk@uvt.nl (Etienne de Klerk), joachim.dahl@mosek.com (Joachim Dahl)

This research was done while the corresponding author (Marandi) was working at Tilburg University. 
Lagrangian dual. Lasserre [21] introduced an LP hierarchy that constructs a sequence of lower bounds for $f^{*}$. Using the Krivine positivstellensatz [19], Lasserre showed that under some assumptions the sequence converges to $f^{*}$. In the hope of getting a tighter lower bound, Lasserre, Toh, and Yang [22] extended the LP hierarchy to an SDP one, called the bounded degree sum-of-squares (BSOS) hierarchy. The advantage of the BSOS hierarchy is that it contains one semi-definite matrix variable, which has a fixed size that is independent of the level of the hierarchy. A major drawback of the BSOS hierarchy lies in the fact that the number of linear variables grows quickly when the level of the hierarchy increases. Also, for a large problem, the size of the semi-definite matrix variable gets large, which makes the hierarchy inefficient. In an effort to resolve these issues, Weisser, Lasserre and Toh 32 introduced a modification of the BSOS hierarchy, called the sparse-BSOS hierarchy, for POPs with a particular structural sparsity, which satisfies the running intersection property (RIP).

The RIP is a well-known concept in graph theory. In the literature of positive semi-definite (PSD) matrices and polynomial optimization, exploiting a sparsity that satisfies the RIP is done by studying the corresponding chordal graphs, see [15 for PSD matrices and 31 for polynomial optimization. The results in [32] can be seen as a combination of the results in the papers [22] and 31 .

POPs have many real-life applications. Some of these applications were studied in the recent paper by Ahmadi and Majumdar 1. In this paper, we analyze the behavior of the sparse-BSOS hierarchy on a class of bilinear programming problems, called pooling problems, and a class of discrete-time optimal control problems.

Solving the pooling problem is attracting considerable interest due to their applications in many real-life optimization problems, like oil refinery planning, chemical process, and water-waste network design. There are many formulations for the pooling problem. Haverly [17, proposed a formulation, called the P-formulation. Even though it was shown by Alfaki and Haugland [3] that the P-formulation problem, in general, is NP-hard, recently Baltean-Lugojan and Misener [6] showed that the three well-known instances proposed by Haverly [17] belong to a subclass of polynomial-time solvable pooling problems. One way of finding a lower bound for the P-formulation 35 problem is by using the McCormick relaxation of each bilinear term, which can be reformulated as a Mixed Integer Linear Programing problem. In order to tighten this relaxation, Tawarmalani and Sahinidis [29] proposed the PQ-formulation. Dey and Gupte [10] proved that even for the 
PQ-formulation, using the McCormick relaxations of the bilinear terms might yield a lower bound that is far from the optimal value of the problem. There are other formulations with different in the surveys by Misener and Floudas [24] and Gupte et al. [16, and the Ph.D. thesis by Alfaki [2].

Recently, semi-definite programming (SDP) hierarchies have been used to find lower bounds for pooling problems. Frimannslund et al. [14] applied the hierarchy proposed by Lasserre 20] to pooling problems. As they pointed out, the fast increase in the sizes of the semi-definite matrix variables in the problem, which is related to the level of the hierarchy, prevents the hierarchy from being applicable for pooling problems. Recently, Marandi, Dahl, and De Klerk 23 evaluated the BSOS hierarchy on pooling problems. They found that the BSOS hierarchy is successful in acquiring the optimal values of small-sized instances, but because of the number of variables, the sparse-BSOS hierarchy on the P-formulation of the pooling problem and compare the results with BSOS.

Another class of problem that we consider in this paper is a class of optimal control problems. A continuous-time optimal control problem finds a control function for a dynamical system such that solving a continuous one by discritizing the time slot that is considered in it. There are different approaches to solve a DTOC problem using a nonlinear optimization problem. In [13, the author provides necessary conditions to make the KKT solutions optimal for the problem. Also, the authors in [9, 11, 31] test their proposed method on a DTOC problem. We refer the reader to the Ph.D. thesis by Nielsen [25] for a more detailed discussion. In this paper, we show how sparse-BSOS hierarchy works on a DTOC problem.

The BSOS and sparse-BSOS hierarchies are applicable to POPs that do not contain any equality constraints. However, all pooling problem formulations and DTOC problems contain many equality constraints. The standard way of dealing with equality constraints is elimination, or replacing them with two inequalities. A way of eliminating equality constraints in the P-formulation was proposed in 23]; however, the elimination may destroy the sparsity pattern. On the other hand, replacing any equality constraints with two inequalities keeps the sparsity pattern but increases the number of constraints in the problem, which is not desirable in the BSOS and sparse-BSOS hierarchies 
because it makes each level harder to solve. In this paper, we show how the hierarchies can be modified to deal with equality constraints directly so that the convergence results remain valid.

The remainder of the paper is organized as follows. Section 2 briefly defines the pooling problem and a formulations of it, namely the P-formulation. In Section 3, we describe continuous- and discrete-time optimal control problems. Section 4 describes the sparse-BSOS hierarchy proposed in 32. Section 5 demonstrates the link between graph theory and polynomial optimization. In we construct a graph corresponding to a POP and exploit a sparsity that satisfies the RIP. In Section 6, we show how to modify the BSOS and sparse-BSOS hierarchies to deal with equality constraints directly. A numerical evaluation of the results is provided in Section 7. This paper contains two appendices. In the first appendix, we construct a toy pooling problem instance to show how eliminating equality constraints of the P-formulation, as proposed in [23], destroys the sparsity that satisfies the RIP. The second appendix contains a table with some extra information regarding our numerical experiments on some DTOC problems.

\section{The $\mathbf{P}$ - formulation of the pooling problem}

In this section, we describe the P-formulation of the pooling problem. The notation we are using is the same as in [16]. A pooling problem is a generalization of a network flow problem in which the inputs possess different specifications. The goal is to minimize the cost of mixing the inputs such that the demand with restrictions over specifications is satisfied. In the pooling problem there are three types of units: ones that store the inputs, which we call inputs and denote by $\mathcal{I}$, ones that mix the incoming flows from inputs, which we call pools and denote by $\mathcal{L}$, and the units that mix the incoming flows from pools and/or inputs, and store the outputs, which we call output and denote by $\mathcal{J}$. The pooling problem can be determined by an acyclic directed graph $G=(\mathcal{N}, \mathcal{A})$, where $N=\mathcal{I} \cup \mathcal{L} \cup \mathcal{J}$, and $\mathcal{A}$ is the set of links between the units, which means $\mathcal{A} \subseteq(\mathcal{I} \times \mathcal{L}) \cup(\mathcal{I} \times \mathcal{J}) \cup(\mathcal{L} \times \mathcal{L}) \cup(\mathcal{L} \times \mathcal{J})$. In this paper, we consider the standard pooling problem, which has no link between the pools and hence $\mathcal{A} \cap \mathcal{L} \times \mathcal{L}=\emptyset$. Figure 1 shows an illustration of a standard pooling problem.

Let $c_{i j}$ be the unit cost of sending one unit of flow from $i$ to $j$. Also, let $y_{i j}$ be the flow-rate between units $i$ and $j$, where the link between the two units has the transmission capacity of at most $u_{i j}$. Also, each unit $i$ may have a capacity $C_{i}$. Let $\mathcal{K}$ be the set of specifications, and let the 


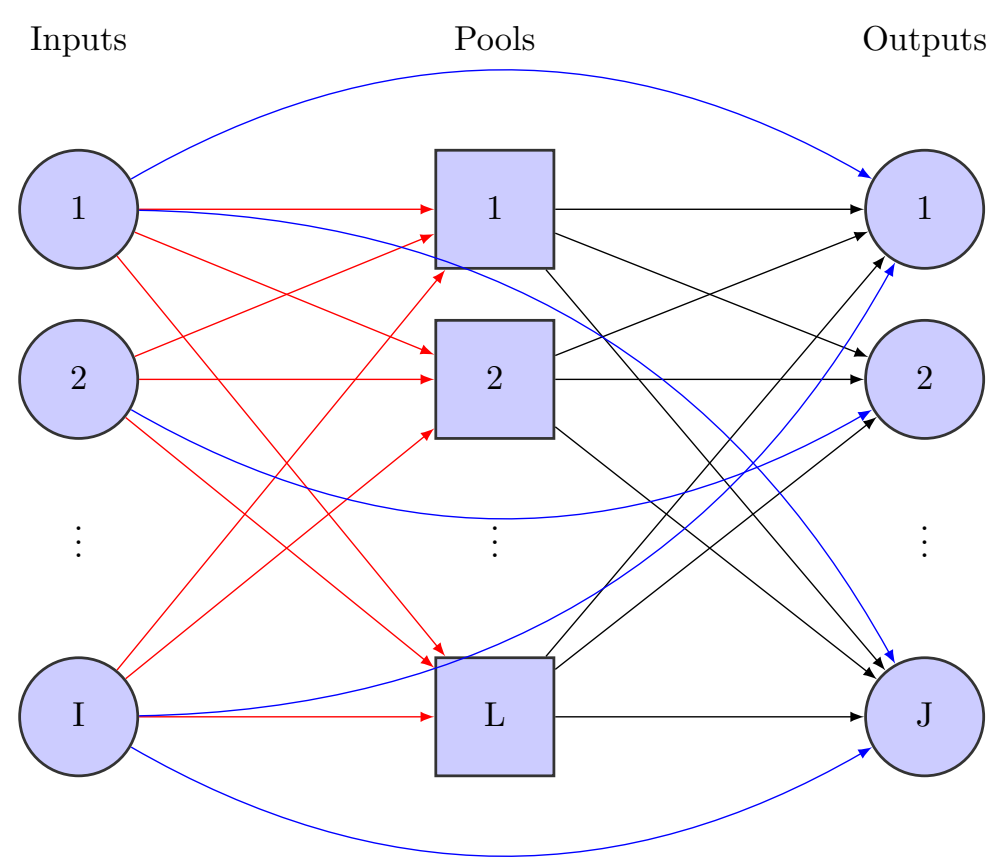

Figure 1: An example of a standard pooling problem with $I$ inputs, $L$ pools and $J$ output.

concentration of the $k$ th specification in input $i$ be $\lambda_{i k}$, and in the pool $l$ be $p_{l k}$. If the concentration in the $j$ th output has the lower and upper bounds restrictions of $\mu_{\min }$ and $\mu_{\max }$, respectively, then the P-formulation of the pooling problem is formulated as follows:

$$
\begin{aligned}
& \min _{y, p} \sum_{(i, j) \in \mathcal{A}} c_{i j} y_{i j} \\
& \text { s.t. } \\
& \sum_{\substack{i \in \mathcal{I}: \\
(i, l) \in \mathcal{A}}} y_{i l}=\sum_{\substack{j \in \mathcal{J}: \\
(l, j) \in \mathcal{A}}} y_{l j}, \quad l \in \mathcal{L}, \\
& \sum_{\substack{j \in \mathcal{C} \cup \mathcal{J}: \\
(i, j) \in \mathcal{A}}} y_{i j} \leq C_{i}, \quad i \in \mathcal{I}, \\
& \sum_{\substack{j \in \mathcal{J}: \\
(l, j) \in \mathcal{A}}} y_{l j} \leq C_{l}, \quad l \in \mathcal{L}, \\
& \sum_{\substack{i \in \mathcal{I} \cup \mathcal{L} \\
(i, j) \in \mathcal{A}}} y_{i j} \leq C_{j}, \quad j \in \mathcal{J}, \\
& 0 \leq y_{i j} \leq u_{i j}, \quad(i, j) \in \mathcal{A},
\end{aligned}
$$




$$
\begin{aligned}
& \sum_{\substack{i \in \mathcal{I} \\
(i, l) \in \mathcal{A}}} \lambda_{i k} y_{i l}=p_{l k} \sum_{\substack{j \in \mathcal{J}: \\
(l, j) \in \mathcal{A}}} y_{l j}, \quad l \in \mathcal{L}, k \in \mathcal{K}, \\
& \sum_{\substack{i \in \mathcal{I}: \\
(i, j) \in \mathcal{A}}} \lambda_{i k} y_{i j}+\sum_{\substack{l \in \mathcal{L}: \\
(l, j) \in \mathcal{A}}} p_{l k} y_{l j} \leq \mu_{j k}^{\max } \sum_{\substack{i \in \mathcal{I} \cup \mathcal{L}: \\
(i, j) \in \mathcal{A}}} y_{i j}, \quad j \in \mathcal{J}, k \in \mathcal{K}, \\
& \sum_{\substack{i \in \mathcal{I} \\
(i, j) \in \mathcal{A}}} \lambda_{i k} y_{i j}+\sum_{\substack{l \in \mathcal{L}: \\
(l, j) \in \mathcal{A}}} p_{l k} y_{l j} \geq \mu_{j k}^{\min } \sum_{\substack{i \in \mathcal{I} \cup \mathcal{L}: \\
(i, j) \in \mathcal{A}}} y_{i j}, \quad j \in \mathcal{J}, k \in \mathcal{K} .
\end{aligned}
$$

Here is a summarized interpretation of the constraints:

(1b): keeping the balance between the total incoming and outgoing flow-rates for each pool,

(1c), (1d), (1e): restricting the units' capacity,

(1f): restricting the capacity of the links between the units,

(1g): keeping the balance of the concentration of each specification in each pool for incoming and outgoing flows,

(1h), (1i): restricting the concentration of each specification in each output by the lower and upper bounds.

In this paper, we use a sparsity pattern and the sparse version of the bounded degree sum-ofsquares hierarchy [32] to solve the P-formulation.

\section{Discrete-time optimal control}

In this section, we briefly describe continuous- and discrete-time optimal control (DTOC) problems. We borrow the notation from [27. A continuous-time optimal control is the optimization problem

$$
\begin{aligned}
\min _{\substack{x(.): \mathbb{R} \rightarrow \mathbb{R}^{n} \\
u(.): \mathbb{R} \rightarrow \mathbb{R}^{m}}} & \int_{0}^{T} F[x(t), u(t), t] d t+S[x(T), T] \\
\text { s.t. } & \dot{x}(t)=f[x(t), u(t), t], \quad \forall t \in[0, T] \\
& x(t) \in \mathcal{X}, u(t) \in \mathcal{U}, \quad \forall t \in[0, T] \\
& x(0)=x_{0}, x(T)=x_{T},
\end{aligned}
$$

where $F[., .,]:. \mathbb{R}^{n} \times \mathbb{R}^{m} \times \mathbb{R} \rightarrow \mathbb{R}$ is a function with continuous partial derivatives with respect to $x(t), S[.,]:. \mathbb{R}^{n} \times \mathbb{R} \rightarrow \mathbb{R}$ is a function with continuous partial derivatives with respect to $x(T)$, 
$f: \mathbb{R}^{n} \times \mathbb{R}^{m} \times \mathbb{R} \rightarrow \mathbb{R}^{m}$ is a function, $\mathcal{X} \subseteq \mathbb{R}^{m}$ and $\mathcal{U} \subseteq \mathbb{R}^{n}$ are given sets, and $x_{0}, x_{T}$ are given vectors.

One of the methods for solving $(2)$ is discretizing the problem and considering the discrete-time optimal control

$$
\begin{aligned}
\min _{\substack{x_{k} \in \mathbb{R}^{n}, k=1, \ldots, N-1 \\
u_{k} \in \mathbb{R}^{m}, k=0, \ldots, N-1}} & \frac{1}{N} \sum_{k=0}^{N-1} F\left[x_{k}, u_{k}, \frac{k T}{N}\right]+S\left[x_{N}, T\right] \\
\text { s.t. } & x_{k+1}=x_{k}+\frac{1}{N} f\left[x_{k}, u_{k}, \frac{k T}{N}\right], k=0, \ldots, N-1 \\
& x_{k} \in \mathcal{X}, k=1, \ldots, N-1, \\
& u_{k} \in \mathcal{U}, k=0, \ldots, N-1,
\end{aligned}
$$

where $x_{0}, x_{N}$ are given. It is clear that when $F$ and $f$ are polynomials, and the sets $\mathcal{X}$ and $\mathcal{U}$ are semi-algebraic, then (3) is a polynomial optimization problem. The size of (3) is related to the number of intervals $(N)$, which can be large. In Section 7.2 , we show how one can apply the sparse-BSOS hierarchy to solve $(3)$.

\section{Sparsity pattern in a polynomial problem}

In this section, we briefly describe the sparse bounded degree sum-of-squares (sparse-BSOS) hierarchy in polynomial optimization introduced, by Weisser, Lasserre and Toh [32]. In what follows, for an integer $m \geq 0$,

$$
[m]:=\left\{\begin{array}{cc}
\{1, \ldots, m\} & \text { if } m>0 \\
\emptyset & \text { if } m=0 .
\end{array}\right.
$$

Also, we assume that $x \in \mathbb{R}^{n}$, and for a given $\mathcal{D} \subseteq[n]$, we denote by $\Sigma[x ; \mathcal{D}]_{\kappa}$, the cone of sum-ofsquares polynomials of degree at most $2 \kappa$, and by $\mathbb{R}[x ; \mathcal{D}]$ the ring of all polynomials in the variables $\left\{x_{i}: i \in \mathcal{D}\right\}$.

For a general polynomial optimization problem

$$
\begin{array}{rl}
f^{*}=\min _{x} & f(x) \\
\text { s.t. } & g_{j}(x) \geq 0, \quad j=1, \ldots, m,
\end{array}
$$

where $x \in \mathbb{R}^{n}, n, m \in \mathbb{N}$ and all $f(x)$ and $g_{j}(x), j=1, \ldots, m$ are $n$-variate polynomials, the running intersection property is defined as follows. 
Definition 1. Problem (4) satisfies the running intersection property (RIP) if there exists $q \in \mathbb{N}$, $\mathcal{D}_{\ell} \subseteq[n]$ and $\mathcal{C}_{\ell} \subseteq[m]$ for all $\ell \in[q]$ such that

- $f=\sum_{\ell=1}^{q} f^{\ell}$, for some $f^{\ell} \in \mathbb{R}\left[x ; \mathcal{D}_{\ell}\right]$, for all $\ell \in[q]$,

- $g_{j} \in \mathbb{R}\left[x ; \mathcal{D}_{\ell}\right]$, for all $j \in \mathcal{C}_{\ell}$, and $\ell \in[q]$,

- $\bigcup_{\ell=1}^{q} \mathcal{D}_{\ell}=[n]$, and $\bigcup_{\ell=1}^{q} \mathcal{C}_{\ell}=[m]$,

- for all $\ell \in[q-1]$, there is an $s \leq \ell$ such that $\left(\mathcal{D}_{\ell+1} \cap \bigcup_{r=1}^{\ell} \mathcal{D}_{r}\right) \subseteq \mathcal{D}_{s}$.

Assume that the running intersection property holds for (4). Let

$$
\mathbb{N}_{d}^{\ell}:=\left\{(\alpha, \beta) \in \mathbb{N}^{2 m}: \quad \alpha_{j}=\beta_{j}=0 \text { if } j \notin \mathcal{C}_{\ell}, \sum_{j \in[m]} \alpha_{j}+\beta_{j} \leq d\right\},
$$

and

$$
h_{\alpha \beta}^{\ell}:=\prod_{j \in[m]} g_{j}^{\alpha_{j}}\left(1-g_{j}\right)^{\beta_{j}} \in \mathbb{R}\left[x, \mathcal{D}_{\ell}\right],(\alpha, \beta) \in \mathbb{N}_{d}^{\ell} .
$$

Then, we have the following result from 32 .

Theorem 1. [32, Theorem 2] Consider the general polynomial optimization problem (4). Suppose that it satisfies the running intersection property, and $g_{j}(x) \leq 1$ for any feasible solution $x, j \in[m]$. Also, assume that for all $\ell \in[q]$, the ring of $\mathbb{R}\left[x ; \mathcal{D}_{\ell}\right]$ is generated by $\left\{1,\left(g_{j}\right)_{j \in \mathcal{C}_{\ell}}\right\}$, and there exists $M_{\ell}>0$ and $j \in \mathcal{C}_{\ell}$ such that $g_{j}=1-\frac{1}{M_{\ell}}\left(\sum_{i \in \mathcal{D}_{\ell}} x_{i}^{2}\right)$. Then, for any fixed $\kappa \in \mathbb{N},\left\{q_{d}^{\kappa}\right\}$ is a non-decreasing sequence and $q_{d}^{\kappa} \rightarrow f^{*}$ as $d \rightarrow+\infty$, where

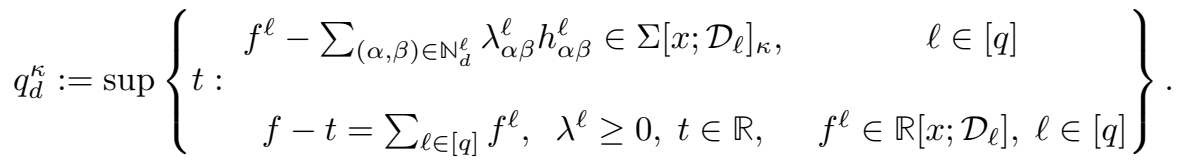

Theorem 1 introduces a non-decreasing sequence that converges to the optimal value of (4) under some assumptions. Instead of (5), we consider the following equivalent problem where the $f^{\ell}, \ell=1, \ldots, q$, have been eliminated,

$$
q_{d}^{\kappa}=\sup \left\{\begin{array}{c}
f-t=\sum_{\ell \in[q]} \sum_{(\alpha, \beta) \in \mathbb{N}_{d}^{\ell}} \lambda_{\alpha \beta}^{\ell} h_{\alpha \beta}^{\ell}+\sum_{\ell \in[q]} \sigma_{\ell} \\
\sigma_{\ell} \in \Sigma\left[x ; \mathcal{D}_{\ell}\right]_{\kappa}, \quad \lambda^{\ell} \geq 0, t \in \mathbb{R}, \ell \in[q]
\end{array}\right\} .
$$


The number of scalar variables in each level of $(6)$ is smaller than the one in the same level of the BSOS hierarchy [22, equation (7)].

Definition 2. Given a vector $b \in \mathbb{R}^{m}(m \leq n)$ and a matrix $A \in \mathbb{R}^{m \times n}$, we call the system of linear constraints $A x=b$ linearly (in)dependent, if the matrix $[A, b] \in \mathbb{R}^{m \times(n+1)}$ has linearly (in)dependent rows.

In the next proposition we show that all constraints in (6) are linearly independent, when the degree of the sums of squares equals to the degree of the whole equality constraint, but before it, we need to emphasize on the following remark.

Remark 1. Let $x \in \mathbb{R}^{n}$ and $p(x)=\sum_{\alpha \in \overline{\mathbb{N}}_{2 \omega}^{[n]}} p_{\alpha} x^{\alpha}$, where

$$
\overline{\mathbb{N}}_{\kappa}^{\mathcal{D}}:=\left\{\alpha \in \mathbb{N}^{n}: \alpha_{i}=0 \text { if } i \notin \mathcal{D}, \sum_{i \in[n]} \alpha_{i} \leq \kappa\right\},
$$

for a set $\mathcal{D} \subseteq[n]$. If $M \in \mathbb{R}\left(\begin{array}{c}n+\omega \\ \omega\end{array}\right) \times\left(\begin{array}{c}n+\omega \\ \omega\end{array}\right)$ is a symmetric matrix variable whose rows (columns) are corresponding to the members of $\overline{\mathbb{N}}_{\omega}^{[n]}$, then linear constraints

$$
p_{\alpha}=\sum_{\substack{\beta, \gamma \in \mathbb{N}[n] \\ \beta+\gamma=\alpha}} M_{\beta \gamma}, \forall \alpha \in \overline{\mathbb{N}}_{2 \omega}^{[n]}
$$

are linearly independent. This is because all the constraints in (7) involve different variables, i.e., no variable appears in two constraints in (7). To see this, let $\beta, \gamma \in \overline{\mathbb{N}}_{\omega}^{[n]}$ be fixed. Due to the construction of the constraints in (7), the variable $M_{\beta \gamma}$ appears only in the constraint corresponding to $\alpha=\beta+\gamma$, and no other constraints.

Proposition 1. Consider problem (4). Let d be such that

$$
2 \kappa=\max \left\{d \max _{j=1, \ldots, m}\left(\operatorname{deg}\left(g_{j}\right)\right), \operatorname{deg}(f), 2 \kappa\right\} .
$$

Then, all equality constraints in (6) are linearly independent, if the polynomial equality is modeled by equating the monomials coefficients.

150 Proof. For each $\ell \in[q]$, set $v^{\ell}=\left(x^{\beta}\right)_{\beta \in \overline{\mathbb{N}}_{\kappa}^{\mathcal{D}_{\ell}}}$. Also, let $\sigma_{\ell}=v^{\ell^{T}} W^{\ell} v^{\ell}$, for each $\ell \in[q]$, where $\left.W^{\ell} \in \mathbb{R}^{\left(n_{\ell}+\kappa\right.}\right) \times\left(\begin{array}{c}n_{\ell}+\kappa \\ \kappa\end{array}\right)$ is a positive semi-definite matrix variable, and $n_{\ell}=\left|\mathcal{D}_{\ell}\right|$. So, Remark 1 implies that the equality constraints in (6) are linearly independent, if the polynomial equality is modeled by equating the monomials coefficients. 
According to the proof of Proposition 1 if $2 \kappa \neq \max \left\{d \max _{j=1, \ldots, m}\left(\operatorname{deg}\left(g_{j}\right)\right), \operatorname{deg}(f), 2 \kappa\right\}$, still

$\operatorname{deg}(f)>\max \left\{d \max _{j=1, \ldots, m}\left(\operatorname{deg}\left(g_{j}\right)\right), 2 \kappa\right\}$, then $\operatorname{deg}(f)=\max \left\{d \max _{j=1, \ldots, m}\left(\operatorname{deg}\left(g_{j}\right)\right), \operatorname{deg}(f), 2 \kappa\right\}$, and clearly the $d$ th iteration of the hierarchy is infeasible, because there is no monomial with degree $\operatorname{deg}(f)$ in $\sum_{\ell \in[q]} \sum_{(\alpha, \beta) \in \mathbb{N}_{d}^{\ell}} \lambda_{\alpha \beta}^{\ell} h_{\alpha \beta}^{\ell}+\sum_{\ell \in[q]} \sigma_{\ell}$.

The main assumption in Theorem 1 is the existence of a splitting that satisfies the running intersection property. So, the question is how to exploit such sparsity for a polynomial optimization problem. In the next section we answer this question.

\section{Polynomial optimization and chordal graphs}

In this section, we study the relation between polynomial optimization and graph theory. Specifically, we mention some results on chordal graphs and use them to exploit sparsity for a polynomial optimization problem that satisfies the running intersection property.

\subsection{Chordal graph and maximal cliques}

In this subsection we recall some well-known results on chordal graphs and maximal cliques. The notation we are using in this section is the same as in 8 .

Definition 3. Consider an undirected graph $G=(V, E)$, where $V$ and $E$ denote the sets of vertices and edges, respectively. A chord of a cycle is any edge joining two nonconsecutive vertices of the cycle. A graph $G$ is called chordal, if every cycle of length greater than 3 has a chord.

Let $\operatorname{adj}(v)$ denote the set of adjacent vertices to a vertex $v$. A vertex ordering $\phi$ of graph $G=(V, E)$ with $n$ vertices is a bijection $\phi: V \rightarrow[n]$, and it can be denoted by indexing the vertex set, such that $V=\left\{v_{1}, \ldots, v_{n}\right\}$, and $\phi\left(v_{i}\right)=i$, for $i \in[n]$. Let $v_{1}, \ldots, v_{n}$ be a vertex ordering of $G$ and set

$$
\mathcal{L}_{i}:=\left\{v_{i}, \ldots, v_{n}\right\}, \quad i \in[n] .
$$

Definition 4. A vertex ordering $\phi$ is a perfect elimination ordering if for any $i \in[n]$ the subgraph of $G$ induced by $\mathcal{L}_{i} \cap \operatorname{adj}\left(v_{i}\right)$ is complete.

Theorem 2. (see, e.g., [8, Theorem 2.2]) A graph $G$ is chordal if and only if $G$ has a perfect 
Definition 5. Let $G=(V, E)$ be any graph. A clique of $G$ is any subset of $V$ for which the induced graph is complete in G. A maximal clique is a clique that is not properly contained in another clique. We denote by $\mathcal{K}_{G}$ the set of all maximal cliques of $G$.

Theorem 3. For a chordal graph $G$, assume that $\left|\mathcal{K}_{G}\right|=q$. Then, there is a labeling $\mathcal{K}_{G}=$ $\left\{\mathcal{K}_{1}, \ldots, \mathcal{K}_{q}\right\}$ such that

$$
\forall \ell \in[q-1], \exists s \leq \ell:\left(\mathcal{K}_{\ell+1} \cap \bigcup_{r=1}^{\ell} \mathcal{K}_{r}\right) \subseteq \mathcal{K}_{s} .
$$

Proof. By invoking [8, Theorem 3.1] and [8, Corollary 1], one can deduce the theorem.

For any vertex ordering $\phi$ of graph $G=(V, E)$, let us add extra edges to $G$ in order to make all $\mathcal{L}_{i} \cap \operatorname{adj}\left(v_{i}\right)$ complete, $i \in[n]$, and denote by $E_{\phi}^{*}$ the union of $E$ with the extra edges. Then clearly $G^{*}=\left(V, E_{\phi}^{*}\right)$ is chordal, because $\phi$ is a perfect elimination ordering for $G^{*}$. The graph $G^{*}$ is called a chordal extension (or triangulation) of $G$.

It is well-known that the Laplacian matrix of a graph $G=(V, E)$, denoted by $L$, is positive semidefinite. We denote the permuted Laplacian matrix of a graph $G$ according to a vertex ordering $\phi$ by $L_{\phi}$. As it is proved in [30, Section 9.1], using the Cholesky factorization $L_{\phi}=R^{T} R$, the nonzero entries of $R+R^{T}$ correspond to the edges in $E_{\phi}^{*}$.

A vertex ordering that minimizes $\left|E_{\phi}^{*} \backslash E\right|$ over all possible vertex orderings of $G$ is called a minimum ordering. Finding a minimum ordering is known to be NP-complete [30, Section 6.6]. There are many polynomial-time algorithms to find a "good" ordering, see [30, Section 6.6]. Minimum degree ordering is such an algorithm, which finds the vertex $v$ with the least degree, set $\phi(v)=i$ and delete $v$ from the graph $G$ in the $i$ th iteration. Finding the vertex with the least degree in each iteration is time-consuming. Therefore, in our numerical results we use the approximate minimum degree ordering (AMD) introduced in [4, which finds upper bounds of the vertices' degree in each iteration and use them to select the vertex $v$.

\subsection{Exploiting sparsity in a polynomial optimization using chordal graphs}

In this subsection, we construct a graph corresponding to problem (4) and use the results mentioned in Section 5.1 to exploit sparsity that satisfies the running intersection property. The graph is essentially the same as the one constructed in [31].

Consider a general polynomial problem (4). A graph $G=(V, E)$ associated to this problem can be constructed as follows: 
- the vertex set $V:=\left\{x_{1}, \ldots, x_{n}\right\}$,

- $E_{j}:=\left\{\left(x_{i}, x_{k}\right):\right.$ variables $x_{i}, x_{k}$ are present in the definition of $\left.g_{j}(x)\right\}, j \in[m]$,

- $E_{0}:=\left\{\left(x_{i}, x_{k}\right):\right.$ product $x_{i} x_{k}$ is present in the definition of $\left.f(x)\right\}$,

- the edge set $E:=\bigcup_{j=0}^{m} E_{j}$.

Let $L$ be the Laplacian matrix of $G=(V, E)$. Using the results in Section 5.1, in order to find the sparsity pattern for the polynomial optimization that satisfies the running intersection property, one can use Algorithm 1

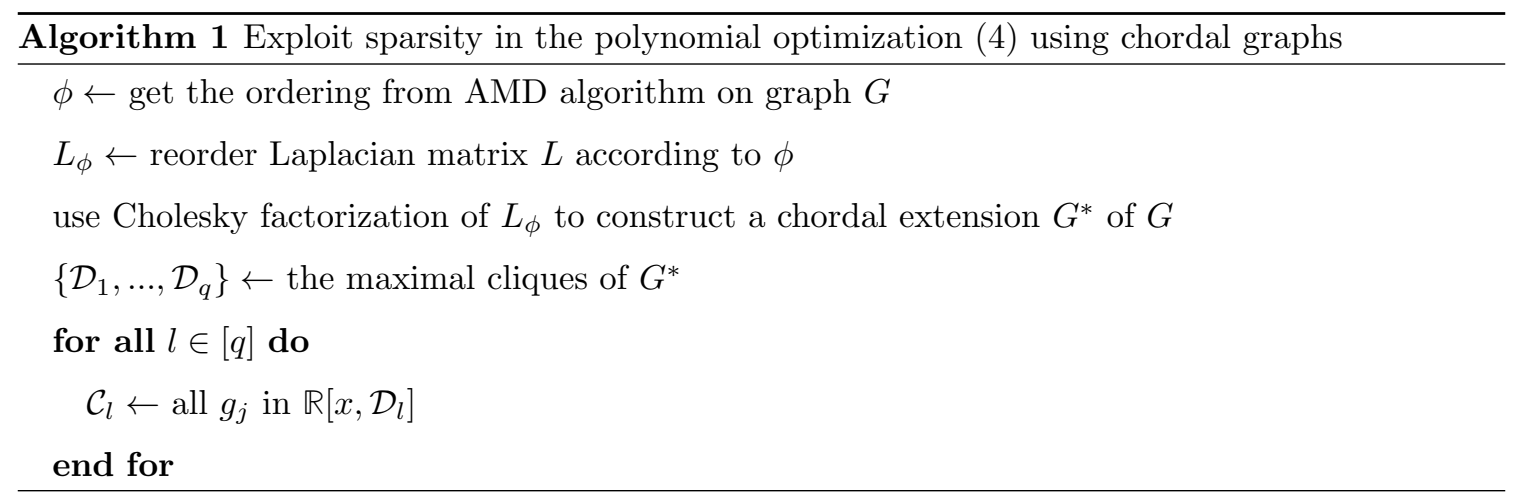

Theorem 4. There is a bijection $\Gamma:[q] \rightarrow[q]$, such that the index blocks $\mathcal{D}_{\Gamma(\ell)}$ and constraint blocks $\mathcal{C}_{\Gamma(\ell)}, \ell \in[q]$, constructed by Algorithm 1 satisfy the running intersection property.

Proof. By Theorem 3 , there is a bijection $\Gamma:[q] \rightarrow[q]$ such that

$$
\forall \Gamma(\ell) \in[q-1], \exists \Gamma(s) \leq \Gamma(\ell):\left(\mathcal{D}_{\Gamma(\ell)+1} \cap \bigcup_{r=1}^{\ell} \mathcal{D}_{\Gamma(r)}\right) \subseteq \mathcal{D}_{\Gamma(s)} .
$$

Now, we show that $f=\sum_{\ell=1}^{q} f^{\ell}$, where $f^{\ell} \in \mathbb{R}\left[x ; \mathcal{D}_{\Gamma(\ell)}\right]$, for all $\ell \in[q]$. Because $f$ is a polynomial, it is sufficient to show that for each monomial in the definition of $f$ there is an $\ell$ such that the monomial belongs to $\mathbb{R}\left[x ; \mathcal{D}_{\ell}\right]$. Let $x^{\beta}$ be a monomial in the definition of $f$, where $\beta \in \mathbb{N}^{n}$. Due to the structure of $E_{0}$, the graph induced by the vertices corresponding to $x^{\beta}$ is complete and hence contained in one of the maximal cliques of $G^{*}$. Therefore, there is an $\ell \in[q]$ such that $x^{\beta} \in \mathbb{R}\left[x ; \mathcal{D}_{\ell}\right]$.

By the construction of $\mathcal{C}_{l}$, it is clear that $g_{j} \in \mathbb{R}\left[x, \mathcal{D}_{\ell}\right]$ for all $j \in \mathcal{C}_{l}, l \in[q]$, and $\bigcup_{\ell=1}^{q} \mathcal{C}_{\ell}=[m]$. Also, $\bigcup_{\ell=1}^{q} \mathcal{D}_{\ell}=[n]$, because $\left\{\mathcal{D}_{1}, \ldots, \mathcal{D}_{q}\right\}$ is the set of all maximal cliques of $G^{*}$. 
In Theorem 4, we showed that there is an ordering of $[q]$ with which the blocks $\mathcal{D}_{\ell}$ and $\mathcal{C}_{\ell}, \ell \in[q]$ satisfy the running intersection property. In the following lemma, we show that we do not need to know the ordering to solve problem (6).

Lemma 1. Let $\Gamma:[q] \rightarrow[q]$ be a bijection for some $q \in \mathbb{N}, \mathcal{D}_{\ell} \subseteq[n]$ and $\mathcal{C}_{\ell} \subseteq[m]$ for all $\ell \in[q]$. Then for each $\kappa, d \in \mathbb{N}, q_{d}^{\kappa}$ in (6) is given by

$$
q_{d}^{\kappa}=\sup \left\{\begin{array}{c}
f-t=\sum_{\ell \in[q]} \sum_{(\alpha, \beta) \in \mathbb{N}_{d}^{\Gamma(\ell)}} \lambda_{\alpha \beta}^{\Gamma(\ell)} h_{\alpha \beta}^{\Gamma(\ell)}+\sum_{\ell \in[q]} \sigma_{\Gamma(\ell)} \\
\sigma_{\ell} \in \Sigma\left[x ; \mathcal{D}_{\ell}\right]_{\kappa}, \quad \lambda^{\ell} \geq 0, t \in \mathbb{R}, \ell \in[q]
\end{array}\right\} .
$$

Proof. The summations in (8) are over $\ell \in[q]$, and may change the order of summations. In other words:

$$
\begin{aligned}
& \sum_{\ell \in[q]} \sum_{(\alpha, \beta) \in \mathbb{N}_{d}^{\Gamma(\ell)}} \lambda_{\alpha \beta}^{\Gamma(\ell)} h_{\alpha \beta}^{\Gamma(\ell)}=\sum_{\ell \in[q]} \sum_{(\alpha, \beta) \in \mathbb{N}_{d}^{\ell}} \lambda_{\alpha \beta}^{\ell} h_{\alpha \beta}^{\ell}, \\
& \sum_{\ell \in[q]} \sigma_{\Gamma(\ell)}=\sum_{\ell \in[q]} \sigma_{\ell} .
\end{aligned}
$$

Theorems 1, 4 and Lemma 1 show that if:

- $g_{j}(x) \leq 1$ for any feasible solution $x, j \in[m]$,

- for all $\ell \in[q]$, the ring of $\mathbb{R}\left[x ; \mathcal{D}_{\ell}\right]$ is generated by $\left\{1,\left(g_{j}\right)_{j \in \mathcal{C}_{\ell}}\right\}$,

- there exists $M_{\ell}>0$ and $j \in \mathcal{C}_{\ell}$ such that $g_{j}=1-\frac{1}{M_{\ell}}\left(\sum_{i \in \mathcal{D}_{\ell}} x_{i}^{2}\right)$,

then for a fixed $\kappa \in \mathbb{N},\left\{q_{d}^{\kappa}\right\}$ is a non-decreasing sequence that converges to the optimal value of (4), when $\mathcal{D}_{\ell}$ and $\mathcal{C}_{\ell}$ are the outputs of Algorithm 1 .

The result of this section can be applied to the P-formulation (1) by elimination of equality constraints proposed in 23]. The following example shows how Algorithm 1 works for "Haverly1" and "Adhya1", two pooling problem instances, after elimination of the equality constraints.

Example 1. "Haverly1" is a pooling problem instance with 3 inputs, 2 outputs and 1 pool, where the inputs are characterized with only 1 specification. The formulation of this instance after elimination of the equality constraints, proposed in [23, Section 3.2], is

$$
\min \quad-200 x_{2}\left(15 x_{1}-12\right)-200 x_{3}\left(15 x_{1}-6\right)+200 x_{4}-1000 x_{5}
$$




$$
\begin{aligned}
\text { s.t. } \quad 1 & \geq-\frac{3}{4}\left(x_{1}-1\right)\left(x_{2}+x_{3}\right) \geq 0 \\
1 & \geq \frac{1}{4}\left(3 x_{1}-1\right)\left(x_{2}+x_{3}\right) \geq 0 \\
1 & \geq 1-2\left(x_{2}+x_{4}\right) \geq 0 \\
1 & \geq 1-\left(x_{3}+x_{5}\right) \geq 0 \\
1 & \geq \frac{1}{2}\left(x_{4}+x_{2}\right)-\frac{2}{5} x_{4}-\frac{3}{5} x_{1} x_{2} \geq 0 \\
1 & \geq \frac{1}{2}\left(x_{5}+x_{3}\right)-\frac{2}{3} x_{5}-x_{1} x_{3} \geq 0 \\
1 & \geq x_{i} \geq 0, \quad i=1, \ldots, 5 .
\end{aligned}
$$

For this problem, the Laplacian matrix corresponding to its graph $G$ (Figure 2a) is

$$
L=\left[\begin{array}{ccccc}
4 & -1 & -1 & -1 & -1 \\
-1 & 3 & -1 & -1 & 0 \\
-1 & -1 & 3 & 0 & -1 \\
-1 & -1 & 0 & 2 & 0 \\
-1 & 0 & -1 & 0 & 2
\end{array}\right]
$$

The output of the AMD algorithm is $\phi\left(\left\{x_{1}, x_{2}, x_{3}, x_{4}, x_{5}\right\}\right)=[5,3,1,4,2]$. Using the Cholesky factorization, one finds out that there is no need to add any extra edge, so $G$ is chordal with the maximal cliques $\mathcal{D}_{1}=\left\{x_{1}, x_{2}, x_{3}\right\}, \mathcal{D}_{2}=\left\{x_{1}, x_{2}, x_{4}\right\}$, and $\mathcal{D}_{3}=\left\{x_{1}, x_{3}, x_{5}\right\}$. Hence,

$$
\begin{aligned}
& \left.\left.\mathcal{C}_{1}=\left\{(9 a), 9 b,(9 g)_{1}, 9 g\right)_{2}, 9 g\right)_{3}\right\}, \\
& \left.\left.\left.\mathcal{C}_{2}=\{9 c, 9 g, 9 g)_{1}, 9 g\right)_{2}, 9 g\right)_{4}\right\}, \\
& \left.\left.\mathcal{C}_{3}=\left\{9 d,, 9 f,(9 g)_{1}, 9 g\right)_{3}, 9 g\right)_{5}\right\},
\end{aligned}
$$

where $[9 g]_{i}$ is the constraint 99 for $x_{i}, i=1, \ldots, 5$.

"Adhya1" is a pooling problem instance that has 5 inputs, 4 outputs, 2 pools where the inputs are characterized with 4 specifications. After elimination of the equality constraints, the problem contains 11 variables and 41 constraints. The graph in Figure 26 shows $G$ where the red dashed arcs are corresponding to the components of $R+R^{T}$ that are zeros in $L_{\phi}$. This means that the red dashed arcs are added to make the graph chordal. For $G^{*}$, the maximal cliques are

$$
\begin{aligned}
& \mathcal{D}_{1}=\left\{x_{1}, x_{2}, x_{3}, x_{4}, x_{5}, x_{9}, x_{10}, x_{11}\right\} \\
& \mathcal{D}_{2}=\left\{x_{2}, x_{3}, x_{4}, x_{5}, x_{6}, x_{9}, x_{10}, x_{11}\right\}
\end{aligned}
$$



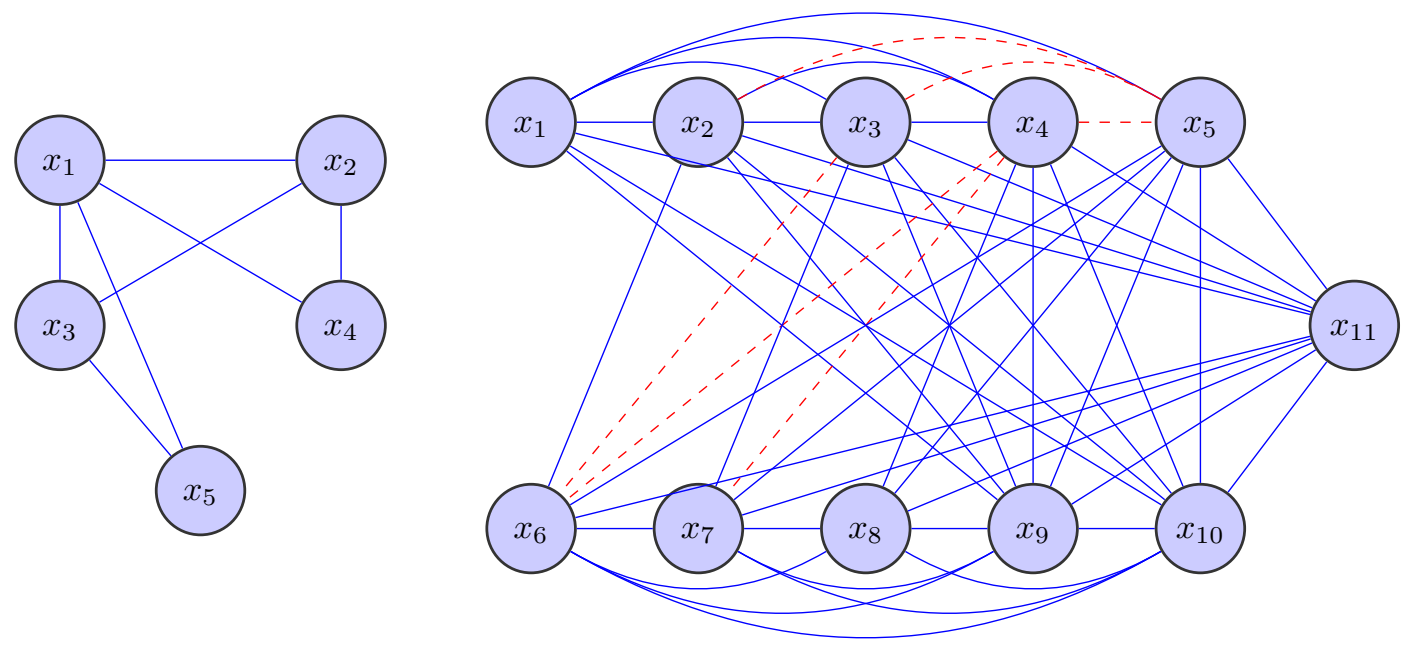

(a) Haverly1

(b) Adhya1

Figure 2: The graphs corresponding to "Haverly1" and "Adhya1" after elimination of the equality constraints. The red dashed arcs are added to make the graph chordal.

$$
\begin{aligned}
& \mathcal{D}_{3}=\left\{x_{3}, x_{4}, x_{5}, x_{6}, x_{7}, x_{9}, x_{10}, x_{11}\right\} \\
& \mathcal{D}_{4}=\left\{x_{4}, x_{5}, x_{6}, x_{7}, x_{8}, x_{9}, x_{10}, x_{11}\right\} .
\end{aligned}
$$

Elimination of the equality constraints may destroy the sparsity pattern that satisfies the running

\section{Problems with equality constraints}

Consider the following algebraic set:

$$
\mathcal{F}:=\left\{x \in \mathbb{R}^{n}: \quad e_{t}(x)=0, t \in[T], \quad g_{i}(x) \geq 0, i \in[m]\right\}
$$


where $e_{t}, g_{i} \in \mathbb{R}[x,[n]], t \in[T], i \in[m]$. In the next theorem we show that one can slightly change the Krivine's positivstellensatz [19] in order to handle algebraic set $\mathcal{F}$.

Theorem 5. Assume that $g_{i}(x) \leq 1$, for all $x \in \mathcal{F}, i \in[m]$. If a polynomial $f(x)$ is positive on $\mathcal{F}$ and the ring of polynomials $\mathbb{R}[x,[n]]$ is generated by $\left\{1, e_{t}(x), g_{i}(x)\right\}_{\substack{i \in[m] \\ t \in[T]}}$, then there is an integer $d$ such that

$$
f(x)=\sum_{(\gamma, \alpha, \theta, \beta) \in \mathbb{N}_{d}^{2 m+2 T}} \lambda_{\gamma, \alpha, \theta, \beta} \prod_{t \in[T]} e_{t}(x)^{\gamma_{t}}\left(1-e_{t}(x)\right)^{\theta_{t}} \prod_{i \in[m]} g_{i}(x)^{\alpha_{i}}\left(1-g_{i}(x)\right)^{\beta_{i}},
$$

for some $\lambda$ such that

$$
\left\{\begin{array}{cc}
\lambda_{\gamma, \alpha, \theta, \beta} \geq 0 & \text { if } \gamma=0 \text { or } T=0, \\
\lambda_{\gamma, \alpha, \theta, \beta} \in \mathbb{R} \quad \text { otherwise. }
\end{array}\right.
$$

Proof. We prove this theorem by induction on $T$, the number of equality constraints. If $T=0$, there is no equality constraints in $\mathcal{F}$. So, the result follows directly from Krivine's positivstellensatz [19]. Now, assume that the result holds for all sets in the form of 10 with $T$ equality constraints and we prove it for a set $\mathcal{F}$ with $T+1$ equality constraints.

Setting $g(x):=e_{T+1}(x)$, we can write $\mathcal{F}$ as follows:

$$
\left\{x: \quad g(x) \geq 0, \quad-g(x) \geq 0, \quad g_{i}(x) \geq 0, i=1, \ldots, m, \quad e_{t}(x)=0, t=1, \ldots, T\right\} .
$$

So, by the induction hypothesis, there is an integer $d$ such that

$$
f(x)=\sum_{\substack{\bar{\alpha}=\left(a_{0}, a_{1}, \alpha, \gamma\right) \\ \bar{\beta}=\left(b_{0}, b_{1}, \beta, \theta\right) \\ \lambda_{\bar{\alpha}, \bar{\beta}} \in \mathbb{N}_{d}^{2 m+2 T+4}}} \lambda_{\bar{\alpha}, \bar{\beta}} g(x)^{a_{0}}(-g(x))^{a_{1}}(1+g(x))^{b_{1}} h_{b_{0} \alpha \beta \gamma \theta}(x),
$$

where,

$$
h_{b_{0} \alpha \beta \gamma \theta}(x)=(1-g(x))^{b_{0}} \prod_{i=1}^{m} g_{i}(x)^{\alpha_{i}}\left(1-g_{i}(x)\right)^{\beta_{i}} \prod_{t=1}^{T} e_{t}(x)^{\gamma_{t}}\left(1-e_{t}(x)\right)^{\theta_{t}},
$$

and

$$
\left\{\begin{array}{lc}
\lambda_{\bar{\alpha}, \bar{\beta}} \geq 0 & \gamma=0 \text { or } T=0 \\
\lambda_{\bar{\alpha}, \bar{\beta}} \in \mathbb{R} & \text { otherwise. }
\end{array}\right.
$$

So, $f(x)$ can be written as

$$
\sum_{\substack{\bar{\alpha}=\left(a_{0}, a_{1}, \alpha, \gamma\right) \\ \bar{\beta}=\left(b_{0}, b_{1}, \beta, \theta\right) \\ \lambda_{\bar{\alpha}, \bar{\beta}} \in \mathbb{N}_{d}^{2 m+2 T+4}}}(-1)^{a_{1}} \lambda_{\bar{\alpha}, \bar{\beta}} g(x)^{a_{0}+a_{1}}(1+g(x))^{b_{1}} h_{b_{0} \alpha \beta \gamma \theta}(x) .
$$


By using binomial theorem for $(1+g(x))^{b_{1}}$, we have

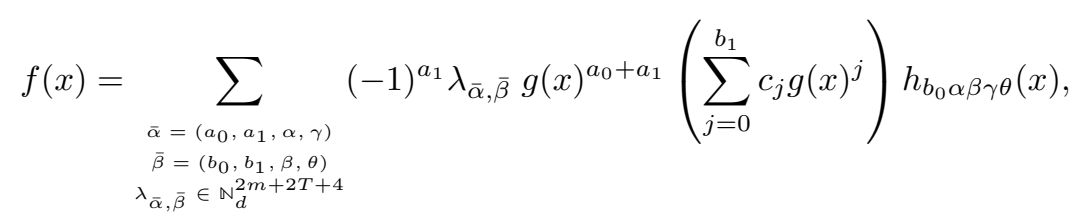

where $c_{j}, j=0, \ldots, b_{1}$, are the binomial coefficients and therefore positive. This means that

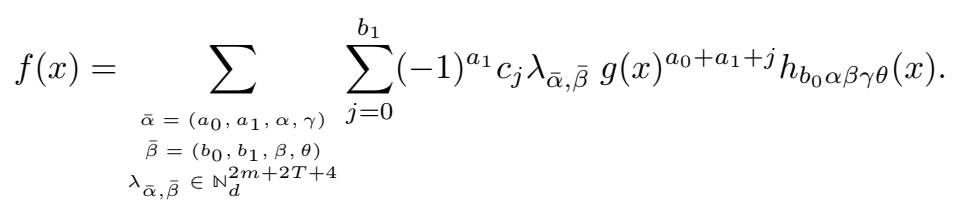

Let us fix $b_{0}, \alpha, \beta, \gamma, \theta$ such that $k:=b_{0}+|\alpha|+|\beta|+|\gamma|+|\theta| \leq d$, and set

$$
\chi_{b_{0}, \alpha, \beta, \gamma, \theta}(x):=\sum_{\left(a_{0}, a_{1}, b_{1}\right) \in \mathbb{N}_{d-k}^{3}} \sum_{j=0}^{b_{1}}(-1)^{a_{1}} c_{j} \lambda_{\bar{\alpha}, \bar{\beta}} g(x)^{a_{0}+a_{1}+j} .
$$

The coefficient of $g(x)^{l}, l=0, \ldots, d-k$, in $\chi_{b_{0}, \alpha, \beta, \gamma, \theta}(x)$ is the summation of some $c_{j} \lambda_{\bar{\alpha}, \bar{\beta}}$ and $-c_{j} \lambda_{\bar{\alpha}, \bar{\beta}}$ corresponding to different $\bar{\beta}$ and $j$. If $l=0$ and $\gamma=0$, or $l=0$ and $T=0$, then, $a_{0}=a_{1}=j=0$, which means the coefficient of $g(x)^{0}$ is nonnegative. Hence,

$$
\begin{aligned}
f(x) & =\sum_{\substack{\left(b_{0}, \alpha, \beta, \gamma, \theta\right) \\
k \leq d}} \chi_{b_{0}, \alpha, \beta, \gamma, \theta}(x) h_{b_{0}, \alpha, \beta, \gamma, \theta}(x) \\
& =\sum_{\substack{\left(b_{0}, \alpha, \beta, \gamma, \theta\right) \\
k \leq d}} \sum_{l=0}^{d-k} \bar{\lambda}_{l, b_{0}, \alpha, \beta, \gamma, \theta} g(x)^{l} h_{b_{0}, \alpha, \beta, \gamma, \theta}(x),
\end{aligned}
$$

for some $\bar{\lambda}$ with real components such that $\bar{\lambda}_{l, b_{0}, \alpha, \beta, \gamma, \theta}$ is nonnegative if $l=0$ and $\gamma=0$ or $l=0$ and $T=0$. So, combining the two summations completes the proof.

Theorem 5 asserts that the coefficients corresponding to the polynomial-multiplications

$$
\prod_{t \in[T]} e_{t}(x)^{\gamma_{t}}\left(1-e_{t}(x)\right)^{\theta_{t}} \prod_{i \in[m]} g_{i}(x)^{\alpha_{i}}\left(1-g_{i}(x)\right)^{\beta_{i}},
$$

with $\gamma \neq 0$, are unrestricted.

Remark 2. Applying Theorem 5 to [32, Theorem 1], if a polynomial optimization problem with 265 feasible region (10) satisfies the assumptions of Theorem 1 1 , then the parts of linear variable $\lambda^{l}$ in (5) associated with the polynomial-multiplications containing equality constraints are unrestricted, and all of the convergence results in [32] are valid. 
Remark 3. Considering Theorem 5 and Remark 2 , one can easily construct the corresponding graph to any polynomial optimization problem with some equality constraints, and exploit the sparsity that satisfies the running intersection property, as described in Section 5.

For a pooling problem, let denote by $G$ the graph of the P-formulation (1) constructed with the procedure in Section 5.2. All nodes in $G$ are corresponding to a variable in the P-formulation (1). Because of the constraint (1c), nodes corresponding to $y_{i l}, y_{i j},(i, l),(i, j) \in \mathcal{A}$ are connected in $G$, for each $i \in \mathcal{I}$. We denote by $\mathcal{K}_{i}, i \in \mathcal{I}$, this type of cliques. The nodes corresponding to $y_{i j}, y_{l j}$, $(i, j),(l, j) \in \mathcal{A}$, for each $j \in \mathcal{J}$ are connected because of $[1 e]$, and we denote the cliques by $\mathcal{K}_{j}$, $j \in \mathcal{J}$. In the same way because of $(1 \mathrm{~d})$, the nodes $y_{i l}, y_{l j},(i, l),(l, j) \in \mathcal{A}$, make the cliques $\mathcal{K}_{l}$ for each $l \in \mathcal{L}$. If there is an $\operatorname{arc}$ in $\mathcal{A}$ between two units, then their corresponding cliques have a node in common. This means that the overlaps between the cliques in $G$ are related to the $\operatorname{arcs}$ in $\mathcal{A}$. Let denote by $\bar{G}$ the network (Figure 1 ) of the pooling problem. The latter discussion shows that the more sparse is $\bar{G}$, the fewer overlaps are between the cliques in $G$. This means that if $\bar{G}$ is sparse then the possibility that in the sparse-BSOS hierarchy the matrix variables have fewer overlaps is high and therefore in this case each level of the sparse-BSOS hierarchy can be solved faster than the same level of the BSOS one.

\section{Numerical result}

The results in Sections 5 and 6 have been implemented in a Julia 0.5 package called "Polyopt", available on https://github.com/MOSEK/Polyopt.jl

In the implementation of the BSOS hierarchy, we model the polynomial equality by equating the monomials coefficients. To construct problems that satisfy the assumptions of Theorem 1 w we add to the problems the constraints

$$
1-\frac{1}{M_{\ell}}\left(\sum_{i \in \mathcal{D}_{\ell}} x_{i}^{2}\right) \geq 0 \quad \ell \in[q] .
$$

All computations in this paper were carried out with Julia 0.5 on an Intel i7-4790 3.60GHz Windows computer with $16 \mathrm{~GB}$ of RAM.

The rest of this section is split into two parts, each of which presents the evaluation of our results on a class of optimization problems. In all tables, \#var., \#const., and bold numbers refer to the number of linear variables, the number of constraints, and the optimal values of the instances 
respectively. Furthermore, we use " $\approx$ " whenever MOSEK cannot solve the problem to optimality and provides us a near optimal solution.

\subsection{The evaluation on the pooling problems using the P-formulation}

In this subsection, we present the numerical evaluation of the sparse-BSOS hierarchy on the P-formulation of the pooling problem instances and compare it with the BSOS hierarchy [22.

Table 1: Detailed information of some pooling problem instances. \# var. and \# const. present the number of variables and constraints in the P-formulation 11, respectively.

* For this instance the exact optimal value is unknown, but the interval on which it lies is known to be [-36233.40, -35812.33].

\begin{tabular}{|c|c|c|c|c|c|c|c|}
\hline & $\begin{array}{c}\text { optimal } \\
\text { value }\end{array}$ & $I$ & $J$ & $L$ & $K$ & \# var. & \# const. \\
\hline Haverly1 & $\mathbf{- 4 0 0 . 0 0}$ & 3 & 2 & 1 & 1 & 7 & 13 \\
\hline Haverly2 & $\mathbf{- 6 0 0 . 0 0}$ & 3 & 2 & 1 & 1 & 7 & 13 \\
\hline Haverly3 & $\mathbf{- 7 5 0 . 0 0}$ & 3 & 2 & 1 & 1 & 7 & 13 \\
\hline Ben-Tal4 & $\mathbf{- 4 5 0 . 0 0}$ & 4 & 2 & 1 & 1 & 8 & 15 \\
\hline Ben-Tal5 & $\mathbf{- 3 , 5 0 0 . 0 0}$ & 5 & 5 & 3 & 2 & 38 & 63 \\
\hline DeyGupte4 & $\mathbf{- 1 . 0 0}$ & 2 & 4 & 2 & 2 & 16 & 58 \\
\hline Foulds2 & $\mathbf{- 1 , 1 0 0 . 0 0}$ & 6 & 4 & 2 & 1 & 22 & 42 \\
\hline Foulds3 & $\mathbf{- 8 . 0 0}$ & 11 & 16 & 8 & 1 & 168 & 235 \\
\hline Foulds4 & $\mathbf{- 8 . 0 0}$ & 11 & 16 & 8 & 1 & 168 & 235 \\
\hline Adhya1 & $\mathbf{- 5 4 9 . 8 0}$ & 5 & 4 & 2 & 4 & 21 & 51 \\
\hline Adhya2 & $\mathbf{- 5 4 9 . 8 0}$ & 5 & 4 & 2 & 6 & 25 & 67 \\
\hline Adhya3 & $\mathbf{- 5 6 1 . 0 5}$ & 8 & 4 & 3 & 6 & 38 & 87 \\
\hline Adhya4 & $\mathbf{- 8 7 7 . 6 0}$ & 8 & 5 & 2 & 4 & 26 & 61 \\
\hline RT2 & $\mathbf{- 4 , 3 9 1 . 8 3}$ & 3 & 3 & 2 & 8 & 32 & 85 \\
\hline sppA0 & Unknown * & 20 & 15 & 10 & 24 & 411 & 1,066 \\
\hline
\end{tabular}

The detailed information of the instances is presented in Table 1 The GAMS files of the instances except DeyGupte4 can be found in the website http://www.ii.uib.no/ mohammeda/ 
spooling/. The instance DeyGupte4 is constructed in [23], and the details can be found in that paper.

In the numerical experiments, we consider $\kappa=1$ in 60. We compare the results of applying the BSOS and sparse-BSOS hierarchies to this formulation when the equality constraints are eliminated [23, and when they are handled directly using Theorem 5. The time in the tables contains the time of constructing the level of the hierarchy and solving it by MOSEK 8.0 [5] in second.

Table 2 presents the results of solving different pooling problem instances with the sparse-BSOS hierarchy (6) and BSOS hierarchy [22].

The comparison has been made in two ways: the columns that are denoted by "with elimination" contain the result of applying the corresponding hierarchy to the pooling problem instances using the elimination method proposed in [23. In the other columns, we use Theorem 5 to handle the equality constraints directly. For a few instances, such as sppA0, the time that is mentioned in Table 2 is larger for the sparse-BSOS hierarchy than the one for the BSOS hierarchy. This is due to the overlap of the matrix variables in the sparse-BSOS hierarchy. The dash "-" in Table 2 means we cannot solve the corresponding level of the hierarchy, due to the size of the problem.

After elimination of equality constraints, the constraints and variables are reduced. This means that in this case, applying Theorem 5 is not worthwhile with respect to the time, because the solver needs to solve a larger problem. Comparing the columns in Tables 2 shows that using Theorem 5 does not necessarily result in better or worse lower bounds. For Adhya4, applying this theorem results in better lower bounds both in the sparse-BSOS and BSOS hierarchies, but this is not the case for Adhya1.

As it can be seen in Tables 2, the sparse-BSOS hierarchy may get worse lower bounds compared to the BSOS hierarchy, which can be seen on the second level of the sparse-BSOS hierarchy of Haverly1-3, Ben-Tal4, Adhya1-3. The advantage of the sparse-BSOS hierarchy is that each level of the hierarchy can be solved relatively faster than the BSOS one, if the problem is sparse. For Foulds2, the lower bounds from the BSOS and sparse-BSOS hierarchies are close but the time in the sparse-BSOS hierarchy is much less than in the BSOS one.

The intuition behind the sparse-BSOS hierarchy is to split the variables into some blocks that contain only some (and not all) variables. If the blocks have many variables in common, then it does not matter much if we merge them together. In Table 3 we present the results of solving the P-formulation of the pooling problem instances when two blocks are merged if the number of 
variables in their intersection is greater than $75 \%$ of the size of the smallest one.

Table 2: Comparing the BSOS hierarchy and sparse-BSOS hierarchies with $\kappa=1$ for the P-formulation. The columns denoted by "with elimination" apply the hierarchy after elimination of equality constraints. The number of maximal cliques $(q)$ in each level is presented between parentheses. The time of the model construction and the solution time is presented between the brackets. Bold-faced entries indicate the (approximate) optimal value.

\begin{tabular}{|c|c|c|c|c|c|}
\hline & iteration & $\begin{array}{l}\text { BSOS with } \\
\text { elimination }\end{array}$ & BSOS & $\begin{array}{l}\text { SBSOS with } \\
\text { elimination }\end{array}$ & SBSOS \\
\hline \multirow{3}{*}{ Haverly1 } & $\mathrm{d}=1$ & $\begin{array}{c}-600.00 \\
{[0.02]}\end{array}$ & $\begin{array}{c}-600.00 \\
{[0.03]}\end{array}$ & $\begin{array}{c}-600.00(3) \\
{[0.01]}\end{array}$ & $\begin{array}{c}-600.00(3) \\
{[0.03]}\end{array}$ \\
\hline & $\mathrm{d}=2$ & $\begin{array}{c}-417.20 \\
{[0.04]}\end{array}$ & $\begin{array}{c}-417.20 \\
{[0.05]}\end{array}$ & $\begin{array}{c}-509.09(3) \\
{[0.02]}\end{array}$ & $\begin{array}{c}-505.00 \\
{[0.04]}\end{array}$ \\
\hline & $\mathrm{d}=3$ & $\begin{array}{c}-400.00 \\
{[0.10]}\end{array}$ & $\begin{array}{c}-400.00 \\
{[0.15]}\end{array}$ & $\begin{array}{c}-400.00(3) \\
{[0.06]}\end{array}$ & $\begin{array}{c}-400.00(3) \\
{[0.07]}\end{array}$ \\
\hline \multirow{4}{*}{ Haverly2 } & $d=1$ & $\begin{array}{c}-1200.00 \\
{[0.03]}\end{array}$ & $\begin{array}{c}-1200.00 \\
{[0.03]}\end{array}$ & $\begin{array}{c}-1200.00(3) \\
{[0.02]}\end{array}$ & $\begin{array}{c}-1200.00(3) \\
{[0.03]}\end{array}$ \\
\hline & $\mathrm{d}=2$ & $\begin{array}{c}-601.67 \\
{[0.05]}\end{array}$ & $\begin{array}{c}-\mathbf{6 0 0 . 0 0} \\
{[0.05]}\end{array}$ & $\begin{array}{c}-1,054.55(3) \\
{[0.05]}\end{array}$ & $\begin{array}{c}-1,054.55(3) \\
{[0.05]}\end{array}$ \\
\hline & $\mathrm{d}=3$ & $\begin{array}{c}-600.00 \\
{[0.16]} \\
\end{array}$ & $\begin{array}{c}-600.00 \\
{[0.21]} \\
\end{array}$ & $\begin{array}{c}-634.65(3) \\
{[0.08]}\end{array}$ & $\begin{array}{c}-628.98(3) \\
{[0.09]}\end{array}$ \\
\hline & $\mathrm{d}=4$ & $\begin{array}{c}-600.00 \\
{[2.63]} \\
\end{array}$ & $\begin{array}{c}-600.00 \\
{[3.28]} \\
\end{array}$ & $\begin{array}{c}-601.09(3) \\
{[0.44]} \\
\end{array}$ & $\begin{array}{c}-600.51(3) \\
{[0.52]} \\
\end{array}$ \\
\hline \multirow{4}{*}{ Haverly3 } & $\mathrm{d}=1$ & $\begin{array}{c}-875.00 \\
{[0.03]}\end{array}$ & $\begin{array}{c}-875.00 \\
{[0.03]}\end{array}$ & $\begin{array}{c}-875.00(3) \\
{[0.03]}\end{array}$ & $\begin{array}{c}-875.00(3) \\
{[0.03]}\end{array}$ \\
\hline & $\mathrm{d}=2$ & $\begin{array}{c}-\mathbf{- 7 5 0 . 0 0} \\
{[0.04]}\end{array}$ & $\begin{array}{c}-\mathbf{- 7 5 0 . 0 0} \\
{[0.05]}\end{array}$ & $\begin{array}{c}-811.98(3) \\
{[0.04]}\end{array}$ & $\begin{array}{c}-810.80(3) \\
{[0.05]}\end{array}$ \\
\hline & $\mathrm{d}=3$ & $\begin{array}{c}-\mathbf{- 7 5 0 . 0 0} \\
{[0.10]}\end{array}$ & $\begin{array}{c}-\mathbf{7 5 0 . 0 0} \\
{[0.16]}\end{array}$ & $\begin{array}{c}-756.95(3) \\
{[0.06]}\end{array}$ & $\begin{array}{c}-754.01(3) \\
{[0.06]}\end{array}$ \\
\hline & $\mathrm{d}=4$ & $\begin{array}{c}-\mathbf{7 5 0 . 0 0} \\
{[1.12]} \\
\end{array}$ & $\begin{array}{c}-\mathbf{7 5 0 . 0 0} \\
{[1.91]} \\
\end{array}$ & $\begin{array}{c}-\mathbf{- 7 5 0 . 0 0}(3) \\
{[0.20]} \\
\end{array}$ & $\begin{array}{c}-\mathbf{- 7 5 0 . 0 0}(3) \\
{[0.31]} \\
\end{array}$ \\
\hline \multirow{3}{*}{ Ben-Tal4 } & $\mathrm{d}=1$ & $\begin{array}{c}-650.00 \\
{[0.03]}\end{array}$ & $\begin{array}{c}-650.00 \\
{[0.03]}\end{array}$ & $\begin{array}{c}-650.00(3) \\
{[0.03]}\end{array}$ & $\begin{array}{c}-650.00(3) \\
{[0.03]}\end{array}$ \\
\hline & $\mathrm{d}=2$ & $\begin{array}{c}-467.20 \\
{[0.05]} \\
\end{array}$ & $\begin{array}{c}-467.20 \\
{[0.05]} \\
\end{array}$ & $\begin{array}{c}-558.29(3) \\
{[0.04]}\end{array}$ & $\begin{array}{c}-540.81(3) \\
{[0.04]} \\
\end{array}$ \\
\hline & $\mathrm{d}=3$ & $\begin{array}{c}-450.00 \\
{[0.18]} \\
\end{array}$ & $\begin{array}{c}-450.00 \\
{[0.22]} \\
\end{array}$ & $\begin{array}{c}-450.00(3) \\
{[0.08]} \\
\end{array}$ & $\begin{array}{c}-450.00(3) \\
{[0.09]}\end{array}$ \\
\hline Ben-Tal5 & $d=1$ & $\begin{array}{c}-\mathbf{3 5 0 0 . 0 0} \\
{[0.06]}\end{array}$ & $\begin{array}{c}-3500.00 \\
{[0.15]}\end{array}$ & $\begin{array}{c}\mathbf{- 3 5 0 0 . 0 0 ( 9 )} \\
{[0.05]} \\
\end{array}$ & $\begin{array}{c}\mathbf{- 3 5 0 0 . 0 0 ( 1 3 )} \\
{[0.09]} \\
\end{array}$ \\
\hline \multirow{3}{*}{ DeyGupte4 } & $d=1$ & $\begin{array}{l}-4.00 \\
{[0.03]}\end{array}$ & $\begin{array}{l}-1.33 \\
{[0.04]}\end{array}$ & $\begin{array}{c}-4.00(1) \\
{[0.04]}\end{array}$ & $\begin{array}{c}-1.33(6) \\
{[0.05]}\end{array}$ \\
\hline & $\mathrm{d}=2$ & $\begin{array}{l}-3.86 \\
{[0.19]}\end{array}$ & $\begin{array}{l}-1.33 \\
{[0.20]}\end{array}$ & $\begin{array}{c}-3.86(1) \\
{[0.19]}\end{array}$ & $\begin{array}{c}-1.33(6) \\
{[0.18]}\end{array}$ \\
\hline & $\mathrm{d}=3$ & $\begin{array}{l}\approx-0.99 \\
{[22.92]}\end{array}$ & $\begin{array}{c}\mathbf{- 1 . 0 0} \\
{[36.77]}\end{array}$ & $\begin{array}{c}\approx-\mathbf{0 . 9 9}(1) \\
{[23.15]}\end{array}$ & $\begin{array}{c}\approx-1.30(6) \\
{[7.29]}\end{array}$ \\
\hline \multirow{3}{*}{ Foulds2 } & $\mathrm{d}=1$ & $\begin{array}{c}-1,200.00 \\
{[0.03]}\end{array}$ & $\begin{array}{c}-1,200.00 \\
{[0.07]}\end{array}$ & $\begin{array}{c}-1,200.00(6) \\
{[0.05]}\end{array}$ & $\begin{array}{c}-1,200.00(8) \\
{[0.05]}\end{array}$ \\
\hline & $\mathrm{d}=2$ & $\begin{array}{c}-1,191.30 \\
{[0.22]} \\
\end{array}$ & $\begin{array}{c}-1,182.80 \\
{[0.34]} \\
\end{array}$ & $\begin{array}{c}-1,193.92(6) \\
{[0.15]}\end{array}$ & $\begin{array}{c}-1,182.80(8) \\
{[0.16]}\end{array}$ \\
\hline & $\mathrm{d}=3$ & $\begin{array}{c}-1,103.10 \\
{[26.45]}\end{array}$ & $\begin{array}{c}\approx-1,102.34 \\
{[101.43]}\end{array}$ & $\begin{array}{c}-1,104.53(6) \\
{[3.79]}\end{array}$ & $\begin{array}{c}-1,103.96(8) \\
{[3.64]}\end{array}$ \\
\hline Foulds3 & $\mathrm{d}=1$ & $\begin{array}{l}\mathbf{- 8 . 0 0} \\
{[62.26]}\end{array}$ & $\begin{array}{c}-8 \\
{[138.25]}\end{array}$ & $\begin{array}{l}-8(20) \\
{[95.30]}\end{array}$ & $\begin{array}{c}\mathbf{- 8}(33) \\
{[136.94]}\end{array}$ \\
\hline
\end{tabular}




\begin{tabular}{|c|c|c|c|c|c|}
\hline Foulds4 & $d=1$ & $\begin{array}{l}\mathbf{- 8 . 0 0} \\
{[61.77]}\end{array}$ & $\begin{array}{c}\mathbf{- 8} \\
{[126.13]}\end{array}$ & $\begin{array}{c}-8(22) \\
{[194.33]}\end{array}$ & $\begin{array}{c}\mathbf{- 8}(33) \\
{[137.44]}\end{array}$ \\
\hline \multirow{3}{*}{ Adhya1 } & $\mathrm{d}=1$ & $\begin{array}{c}-999.32 \\
{[0.0 .3]}\end{array}$ & $\begin{array}{c}-999.32 \\
{[0.05]}\end{array}$ & $\begin{array}{c}-999.32(4) \\
{[0.04]}\end{array}$ & $\begin{array}{c}-999.32(4) \\
{[0.05]}\end{array}$ \\
\hline & $\mathrm{d}=2$ & $\begin{array}{c}-721.12 \\
{[0.19]}\end{array}$ & $\begin{array}{c}-997.63 \\
{[0.40]}\end{array}$ & $\begin{array}{c}-957.02(4) \\
{[0.13]}\end{array}$ & $\begin{array}{c}\approx-998.43(4) \\
{[0.22]}\end{array}$ \\
\hline & $\mathrm{d}=3$ & $\begin{array}{c}-578.27 \\
{[36.50]} \\
\end{array}$ & $\begin{array}{c}\approx-669.86 \\
{[210.43]}\end{array}$ & $\begin{array}{c}-778.72(4) \\
{[3.42]} \\
\end{array}$ & $\begin{array}{c}\approx-860.91(4) \\
{[17.16]}\end{array}$ \\
\hline \multirow{3}{*}{ Adhya2 } & $\mathrm{d}=1$ & $\begin{array}{c}-798.29 \\
{[0.01]} \\
\end{array}$ & $\begin{array}{c}-854.10 \\
{[0.05]}\end{array}$ & $\begin{array}{c}-798.29(4) \\
{[0.01]}\end{array}$ & $\begin{array}{c}-854.10(4) \\
{[0.04]}\end{array}$ \\
\hline & $\mathrm{d}=2$ & $\begin{array}{c}-577.00 \\
{[0.24]} \\
\end{array}$ & $\begin{array}{c}-853.82 \\
{[0.89]} \\
\end{array}$ & $\begin{array}{c}-686.60(4) \\
{[0.12]} \\
\end{array}$ & $\begin{array}{c}-854.10(4) \\
{[0.44]} \\
\end{array}$ \\
\hline & $d=3$ & $\begin{array}{c}-566.52 \\
{[39.02]} \\
\end{array}$ & $\begin{array}{l}\approx-575.81 \\
{[1,163.57]}\end{array}$ & $\begin{array}{c}-573.31(4) \\
{[8.42]} \\
\end{array}$ & $\begin{array}{c}-749.44(4) \\
{[99.10]}\end{array}$ \\
\hline \multirow{2}{*}{ Adhya3 } & $\mathrm{d}=1$ & $\begin{array}{c}-882.84 \\
{[0.02]} \\
\end{array}$ & $\begin{array}{c}-882.84 \\
{[0.18]} \\
\end{array}$ & $\begin{array}{c}-882.84(5) \\
{[0.03]} \\
\end{array}$ & $\begin{array}{c}-882.84(7) \\
{[0.13]} \\
\end{array}$ \\
\hline & $\mathrm{d}=2$ & $\begin{array}{c}-805.08 \\
{[0.64]} \\
\end{array}$ & $\begin{array}{c}-882.73 \\
{[9.28]} \\
\end{array}$ & $\begin{array}{c}-870.55(5) \\
{[0.31]}\end{array}$ & $\begin{array}{c}-882.84(7) \\
{[3.20]}\end{array}$ \\
\hline \multirow{3}{*}{ Adhya4 } & $d=1$ & $\begin{array}{c}-1055.00 \\
{[0.01]}\end{array}$ & $\begin{array}{c}-1003.33 \\
{[0.05]}\end{array}$ & $\begin{array}{c}-1055.00(5) \\
{[0.02]}\end{array}$ & $\begin{array}{c}-1003.33(7) \\
{[0.05]}\end{array}$ \\
\hline & $\mathrm{d}=2$ & $\begin{array}{c}-1,040.00 \\
{[0.33]}\end{array}$ & $\begin{array}{c}-1003.33 \\
{[0.90]} \\
\end{array}$ & $\begin{array}{c}-1,040.00(5) \\
{[0.20]}\end{array}$ & $\begin{array}{c}-1003.33(7) \\
{[0.38]}\end{array}$ \\
\hline & $\mathrm{d}=3$ & $\begin{array}{c}\approx-908.13 \\
{[317.94]}\end{array}$ & $\begin{array}{c}-893.68 \\
{[1,343.85]} \\
\end{array}$ & $\begin{array}{c}-1,012.16(5) \\
{[17.46]}\end{array}$ & $\begin{array}{c}-982.42(7) \\
{[40.42]} \\
\end{array}$ \\
\hline \multirow{2}{*}{ RT2 } & $\mathrm{d}=1$ & $\begin{array}{c}-45,420.50 \\
{[0.02]} \\
\end{array}$ & $\begin{array}{c}-22,578.70 \\
{[0.20]}\end{array}$ & $\begin{array}{c}-45,420.49 \\
{[0.02]} \\
\end{array}$ & $\begin{array}{c}-22,578.70(13) \\
{[0.22]}\end{array}$ \\
\hline & $\mathrm{d}=2$ & $\begin{array}{c}\approx-39,287.34 \\
{[0.54]}\end{array}$ & $\begin{array}{c}-22,153.45 \\
{[3.42]} \\
\end{array}$ & $\begin{array}{c}\approx-39,291.55(2) \\
{[0.56]}\end{array}$ & $\begin{array}{c}-22,153.48(13) \\
{[1.66]}\end{array}$ \\
\hline sppA0 & $\mathrm{d}=1$ & $\begin{array}{c}-47,675.00 \\
{[193.38]} \\
\end{array}$ & - & $\begin{array}{c}-47,675.00(27) \\
{[413.88]} \\
\end{array}$ & - \\
\hline
\end{tabular}

For Haverly1-3, and Ben-Tal4, there is no block merging. For DeyGupte4, Foulds3-4, Adhya2-3, RT2 and sppA0, all of the blocks get merged, which results in the BSOS hierarchy. Hence, we present the results for the rest of the instances in Table 3 . In this table, we present the number of linear variables (the number of $\lambda^{\ell}, \ell \in[q]$ ), the number of constraints, and the size of the semi-definite variables (size of PSDs) as well as the lower bounds that we get in each level of the hierarchies. As one can see, in all the instances in Table 3 , the elimination of the equality constraints results in the high overlap in the maximal cliques of the associated graph to the P-formulation, and therefore we have merged all the maximal cliques. For the instances Foulds2 and Adhya4, even though the number of constraints in the first level of the sparse-BSOS hierarchy is less when the equality constraints are eliminated compared to the one when the equalities are handled directly, it is other way around in the third iteration. This is because, for each $\ell \in[q]$, the constraints in $\mathbb{R}\left[x ; \mathcal{D}_{\ell}\right]$ are fewer than the ones in $\mathbb{R}[y]$, where $x$ is the vector of variables in the P-formulation (1) 
and $y$ is the vector of variables in the P-formulation after eliminating equality constraints.

According to the discussion in Section 6 , the overlaps in the matrix variables of the sparse-BSOS hierarchy corresponding to the P-formulation (1) is related to the sparsity of the network of the pooling problem, Figure 1, and the number of specifications. As one can see, for the four instances in Table 3 the networks of the instances are highly sparse, and therefore the possibility that each level of the sparse-BSOS hierarchy can be solved faster than the same level in the BSOS hierarchy is high. For Adhya2, the network is the same as Adhya1, but because the number of specifications is much higher in Adhya2, the overlaps of the matrix variables in the sparse-BSOS hierarchy are much higher and more than $75 \%$ of their sizes.

Table 3: The result of solving the P-formulation of pooling problem instances with sparse-BSOS hierarchy when two maximal cliques are merged if the intersection size is larger than $75 \%$ of the size of smallest maximal clique (the size(s) of the positive semi-definite matrix variable(s) is (are) presented in the second row of each iteration).

\begin{tabular}{|c|c|c|c|c|c|c|c|}
\hline & \multirow{2}{*}{ iteration } & \multicolumn{3}{|c|}{ SBSOS with elimination } & \multicolumn{3}{|c|}{ SBSOS } \\
\hline & & $\begin{array}{c}\text { Value } \\
([\text { size of PSDs }]) \\
{[\text { time }]}\end{array}$ & \#const. & \#var. & $\begin{array}{c}\text { Value } \\
([\text { size of PSDs }]) \\
{[\text { time }]}\end{array}$ & \#const. & \#var. \\
\hline Ben-Tal5 & $d=1$ & $\begin{array}{c}-3,500.00 \\
([30]) \\
{[0.06]}\end{array}$ & 465 & 112 & $\begin{array}{c}-3,500.00 \\
([12,22,29]) \\
{[0.07]}\end{array}$ & 601 & 169 \\
\hline \multirow{3}{*}{ Foulds2 } & $d=1$ & $\begin{array}{c}-1,200.00 \\
([19]) \\
{[0.02]}\end{array}$ & 190 & 80 & $\begin{array}{c}-1,200.00 \\
([2 \times 8,19]) \\
{[0.02]}\end{array}$ & 220 & 112 \\
\hline & $d=2$ & $\begin{array}{c}-1,191.30 \\
([19]) \\
{[0.17]}\end{array}$ & 973 & 3,161 & $\begin{array}{c}-1,182.80 \\
([2 \times 8,19]) \\
{[0.16]}\end{array}$ & 1,109 & 2,665 \\
\hline & $d=3$ & $\begin{array}{c}-1,103.14 \\
([19]) \\
{[25.97]}\end{array}$ & 13,601 & 85,321 & $\begin{array}{c}\approx-1,102.74 \\
([2 \times 8,19]) \\
{[20.61]}\end{array}$ & 14,609 & 49,529 \\
\hline \multirow{3}{*}{ Adhya1 } & $d=1$ & $\begin{array}{c}-999.32 \\
([12]) \\
{[0.01]}\end{array}$ & 78 & 86 & $\begin{array}{c}-999.32 \\
([12,19]) \\
{[0.04]}\end{array}$ & 223 & 114 \\
\hline & $d=2$ & $\begin{array}{c}-721.12 \\
([12]) \\
{[0.13]} \\
\end{array}$ & 543 & 3,656 & $\begin{array}{c}\approx-998.09 \\
([12,19]) \\
{[0.42]}\end{array}$ & 2,317 & 4,035 \\
\hline & $d=3$ & $\begin{array}{c}-578.27 \\
([12]) \\
{[38.18]}\end{array}$ & 5,105 & 105,996 & $\begin{array}{c}\approx-776.44 \\
([12,19]) \\
{[71.97]}\end{array}$ & 41,127 & 107,300 \\
\hline
\end{tabular}




\begin{tabular}{|c|c|c|c|c|c|c|c|}
\hline \multirow{3}{*}{ Adhya4 } & $\mathrm{d}=1$ & $\begin{array}{c}-1,055.00 \\
([17]) \\
{[0.01]}\end{array}$ & 153 & 106 & $\begin{array}{c}-1,003.33 \\
([2 \times 14,19]) \\
{[0.03]}\end{array}$ & 290 & 156 \\
\hline & $\mathrm{d}=2$ & $\begin{array}{c}-1,040.00 \\
([17]) \\
{[0.33]}\end{array}$ & 1,730 & 5,566 & $\begin{array}{c}-1,003.33 \\
([2 \times 14,19]) \\
{[0.31]}\end{array}$ & 3,253 & 5,194 \\
\hline & $d=3$ & $\begin{array}{c}\approx-908.13 \\
\quad([17]) \\
{[360.27]}\end{array}$ & 27,922 & 198,486 & $\begin{array}{c}-974.55 \\
([2 \times 14,19]) \\
{[62.54]}\end{array}$ & 61,401 & 135,764 \\
\hline
\end{tabular}

\subsection{The evaluation on DTOC problems}

Consider a DTOC problem (3). If $F$ and $f$ are polynomials, and $\mathcal{X}, \mathcal{U}$ are semi-algebraic sets, then it is easy to see that (3) satisfies the RIP with the maximal cliques

$$
\mathcal{D}_{k}=\left\{x_{k+1}, x_{k}, u_{k}\right\}, k=0, \ldots, N-1
$$

In the numerical experiments, we consider the following DTOC problem:

$$
\begin{array}{ll}
\min _{\substack{x_{k} \in \mathbb{R}, k=1, \ldots, N-1 \\
u_{k} \in \mathbb{R}, k=0, \ldots, N-1}} & \frac{1}{N-1} \sum_{k=0}^{N-1}\left(x_{k}^{2}+u_{k}^{2}\right) \\
\text { s.t. } & x_{k+1}=x_{k}+\frac{1}{N}\left(x_{k}^{2}-u_{k}\right), k=0, \ldots, N-1, \\
& x^{\ell} \leq x_{k} \leq x^{u}, k=1, \ldots, N-1, \\
& u^{\ell} \leq u_{k} \leq u^{u}, k=0, \ldots, N-1,
\end{array}
$$
we use AIMMS 4.39 [7]. For a fair comparison, we report the times that MOSEK 8.0 needs to solve the level of the hierarchies, as well as the times taken by BARON and CONOPT to solve the 
DTOC problems in seconds. We put a maximum time limit of 120 seconds for all methods. We emphasize that we do not report the result of using the BSOS hierarchy for this problem, since the number of variables in (11) is large and the size of the semi-definite matrix variable in the BSOS hierarchy makes the hierarchy inefficient for this type of problems.

Table 4: Numerical experiments on DTOC problems 11 with the input data $\left(x^{\ell}, x^{u}, x_{0}, x_{N}, u^{\ell}, u^{u}\right)=$ $(0.9,5,1,1,-10,10)$, and different $N$, for $\kappa=2, d=2$, .

\begin{tabular}{|c|c|c|c|c|c|c|c|c|}
\hline \multirow[b]{2}{*}{$\mathrm{N}$} & \multicolumn{2}{|c|}{$\begin{array}{l}\text { SBSOS with } \\
\text { Theorem } 5\end{array}$} & \multicolumn{2}{|c|}{$\begin{array}{c}\text { SBSOS without } \\
\text { Theorem } 5\end{array}$} & \multicolumn{2}{|c|}{ BARON 28 } & \multicolumn{2}{|c|}{ CONOPT 12$]$} \\
\hline & value & time & value & time & $\begin{array}{c}\text { upper and } \\
\text { lower bounds }\end{array}$ & time & Upper bound & time \\
\hline 50 & 1.6600 & 0.14 & 1.6600 & 0.19 & {$[1.6600,1.6600]$} & 1.06 & 1.6600 & 0.02 \\
\hline 250 & 1.6569 & 0.78 & 1.6569 & 1.06 & {$[1.6544,1.6569]$} & 120.00 & 1.6569 & 0.08 \\
\hline 500 & 1.6566 & 1.65 & 1.6566 & 2.28 & {$[1.6201,1.6566]$} & 120.00 & 1.6566 & 0.19 \\
\hline 1000 & 1.6564 & 3.77 & 1.6564 & 4.88 & {$[1.5932,1.6564]$} & 120.00 & 1.6564 & 0.80 \\
\hline 2000 & 1.6563 & 9.14 & 1.6563 & 12.68 & {$[1.5613,1.6563]$} & 120.00 & 1.6563 & 2.17 \\
\hline 3000 & 1.6563 & 14.54 & 1.6563 & 22.14 & {$[1.5611,1.6563]$} & 120.00 & 1.6563 & 5.23 \\
\hline 4000 & $\approx 1.6563$ & 25.12 & $\approx 1.6563$ & 31.14 & {$[0.8118,1.6562]$} & 120.00 & 1.6562 & 8.77 \\
\hline 5000 & $\approx 1.6562$ & 36.33 & $\approx 1.6562$ & 54.83 & {$[0.8118,1.6562]$} & 120.00 & 1.6562 & 42.20 \\
\hline
\end{tabular}

Table 4 presents the results of applying the SBSOS with and without Theorem 5 to DTOC problems for fixed

$$
\left(x^{\ell}, x^{u}, x_{0}, x_{N}, u^{\ell}, u^{u}\right)=(0.9,5,1,1,-10,10)
$$

and different $N$, and demonstrates the advantage of applying Theorem 5 to the sparse-BSOS hierarchy. As one can see in the table, the lower bounds that we obtain from the SBSOS with and without Theorem 5 are the same but applying this theorem helps us to get the lower bounds much faster. Moreover, the lower bounds are equal to the objective values of the solutions found by both BARON and CONOPT. Therefore, even though the solvers can not guarantee optimality of the solutions, the lower bounds from the hierarchies assure us that the solutions are globally optimal for $N=250,1000,2000,3000$. For $N=4000,5000$ the lower bounds that we get from the hierarchies are not precise and hence we cannot deduce that the solution is optimal; however the lower bounds 
are close to the objective values of the solutions.

Table 5 in Appendix B provides the number of variables, the number of constraints, and the size of the semi-definite variables in the SBSOS with and without Theorem 5

\section{Conclusion}

In this paper, we studied the sparse-BSOS hierarchy introduced in 32 . We first showed how to find a splitting of variables for a general polynomial optimization problem that satisfies the running intersection property. Then, we modified this hierarchy to handle the problems with equality constraints. The results in this paper has been implemented in a Julia 0.5 package "Polyopt" to solve a polynomial optimization problem.

In the numerical results we compared the sparse-BSOS hierarchy with the BSOS one, when Theorem 5 is applied. For the P-formulation, the problems in each level of the sparse-BSOS hierarchy could be solved faster than the BSOS one if the network of the pooling problem is sparse enough and the number of specifications is small, like the instance Foulds2. The quality of the lower bounds we got from the sparse-BSOS hierarchy could sometimes be worse than the BSOS hierarchy. The modification we proposed to the BSOS and sparse-BSOS hierarchies to handle equality constraints could sometimes yield much better lower bounds than the original hierarchies, like the first and second levels of the hierarchies in the DeyGupte4 instance.

Applying the sparse-BSOS hierarchy to the discrete-time optimal control problems showed that the lower bounds we got form the sparse-BSOS hierarchy and the modified one using Theorem 5

were the same, but each level of the modified hierarchy could be solved faster. Also, the lower bounds guaranteed optimality of the solutions found by the solvers BARON and CONOPT.

\section{Acknowledgment}

The authors would like to thank Ruth Misener for bringing the paper [6] to their attention. Also, the authors are grateful to the two anonymous reviewers for their helpful and constructive comments that greatly contributed to improving the paper. 


\section{References}

[1] A. A. Ahmadi and A. Majumdar. Some applications of polynomial optimization in operations research and real-time decision making. Optimization Letters, 10(4):709-729, 2016.

[2] M. Alfaki. Models and solution methods for the pooling problem. Ph.D. thesis, University of Bergen, 2012.

[3] M. Alfaki and D. Haugland. Strong formulations for the pooling problem. Journal of Global Optimization, 56(3):897-916, 2013.

[4] P. R. Amestoy, T. A. Davis, and I. S. Duff. An approximate minimum degree ordering algorithm. SIAM Journal on Matrix Analysis and Applications, 17(4):886-905, 1996.

[5] MOSEK ApS. The MOSEK optimization toolbox for MATLAB manual. Version 8.0 (Revision 83), 2017.

[6] R. Baltean-Lugojan and R. Misener. Piecewise parametric structure in the pooling problem: from sparse strongly-polynomial solutions to NP-hardness. Journal of Global Optimization, pages 1-36, 2017.

[7] J. Bisschop. AIMMS optimization modeling. Paragon Decision Technology, Bellevue, 2006.

[8] J. R. S. Blair and B. Peyton. An Introduction to Chordal Graphs and Clique Trees, pages 1-29. Springer New York, New York, NY, 1993.

[9] Th. F. Coleman and A. Liao. An efficient trust region method for unconstrained discrete-time optimal control problems. Computational Optimization and Applications, 4(1):47-66, 1995.

[10] S. S. Dey and A. Gupte. Analysis of MILP techniques for the pooling problem. Operations Research, 63(2):412-427, 2015.

[11] G. Di Pillo, L. Grippo, and F. Lampariello. A class of structured quasi-newton algorithms for optimal control problems. IFAC Proceedings Volumes, 16(8):101-107, 1983.

[12] A. Drud. CONOPT: A system for large scale nonlinear optimization. Reference manual for CONOPT subroutine library, ARKI Consulting and Development A/S, Bagsvaerd, Denmark, 1996. 
[13] T. L. Friesz. Nonlinear Programming and Discrete-Time Optimal Control, pages 33-78. Springer US, 2010.

[14] L. Frimannslund, M. El Ghami, M. Alfaki, and D. Haugland. Solving the pooling problem with LMI relaxations. S. Cafieri, B.G.-Tóth, E. Hendrix, L. Liberti and F. Messine (Eds), Proceedings of the Toulouse Global Optimization Workshop (TOGO), pages 51-54, 2010.

[15] M. Fukuda, M. Kojima, K. Murota, and K. Nakata. Exploiting sparsity in semidefinite programming via matrix completion I: General framework. SIAM Journal on Optimization, 11(3):647-674, 2001.

[16] A. Gupte, Sh. Ahmed, S. S. Dey, and M. S. Cheon. Relaxations and discretizations for the pooling problem. Journal of Global Optimization, 67(3):631-669, 2017.

[17] C. A. Haverly. Studies of the behavior of recursion for the pooling problem. ACM SIGMAP Bulletin, (25):19-28, 1978.

[18] S. Kim, M. Kojima, and H. Waki. Generalized lagrangian duals and sums of squares relaxations of sparse polynomial optimization problems. SIAM Journal on Optimization, 15(3):697-719, 2005.

[19] J. L. Krivine. Anneaux préordonnés. Journal d'analyse mathématique, 12(1):307-326, 1964.

[20] J. B. Lasserre. Global optimization with polynomials and the problem of moments. SIAM Journal on Optimization, 11(3):796-817, 2001.

[21] J. B. Lasserre. Polynomial programming: LP-relaxations also converge. SIAM Journal on Optimization, 15(2):383-393, 2005.

[22] J. B. Lasserre, K. Toh, and Sh. Yang. A bounded degree SOS hierarchy for polynomial optimization. EURO Journal on Computational Optimization, 5(1):87-117, 2017.

[23] A. Marandi, J. Dahl, and E. de Klerk. A numerical evaluation of the bounded degree sum-ofsquares hierarchy of Lasserre, Toh, and Yang on the pooling problem. Annals of Operations Research, pages 1-26, 2017. 
[24] R. Misener and Ch. A. Floudas. Advances for the pooling problem: modeling, global optimization, and computational studies. Applied and Computational Mathematics, 8(1):3-22, 2009.

[25] I. Nielsen. Structure-Exploiting Numerical Algorithms for Optimal Control. PhD thesis, Linköping University, 2017.

[26] P. M. Pardalos and S. A. Vavasis. Quadratic programming with one negative eigenvalue is NP-hard. Journal of Global Optimization, 1(1):15-22, 1991.

[27] B. Ran and D. Boyce. Discrete optimal control and nonlinear programming. In Modeling Dynamic Transportation Networks, pages 49-67. Springer, 1996.

[28] N. V. Sahinidis. BARON 14.3.1: Global Optimization of Mixed-Integer Nonlinear Programs, User's Manual, 2014.

[29] M. Tawarmalani and N. V. Sahinidis. Convexification and global optimization in continuous and mixed-integer nonlinear programming: theory, algorithms, software, and applications, volume 65. Springer Science \& Business Media, 2002.

[30] L. Vandenberghe and M. S. Andersen. Chordal graphs and semidefinite optimization. Foundations and Trends in Optimization, 1(4):241-433, 2015.

[31] H. Waki, S. Kim, M. Kojima, and M. Muramatsu. Sums of squares and semidefinite program relaxations for polynomial optimization problems with structured sparsity. SIAM Journal on Optimization, 17(1):218-242, 2006.

[32] T. Weisser, J. B. Lasserre, and K. Toh. Sparse-BSOS: a bounded degree SOS hierarchy for large scale polynomial optimization with sparsity. Mathematical Programming Computation, pages $1-32,2017$.

\section{Appendices}

\section{A. New pooling problem instance}

In this appendix, we construct a pooling problem instance with three inputs, two pools, and four outputs. Then, we show the elimination method proposed in [23] destroys the sparsity pattern 


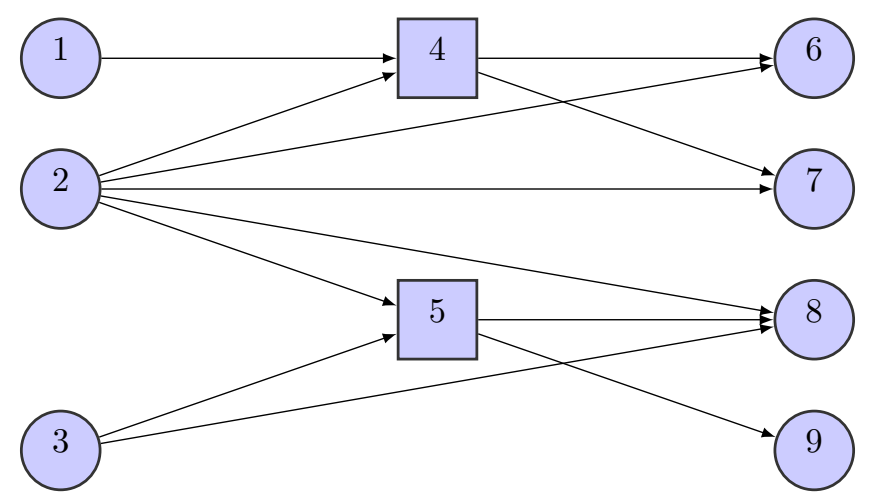

Figure 3: An example of a standard pooling problem for which the elimination method proposed in 23] destroys the sparsity pattern.

that exists in the P-formulation.

Consider the pooling problem instance with the diagram drawn in Figure 3. Assume that each input and pool has a capacity restriction of 600 . The restriction of the capacities of the outputs 6,7 , 8, and 9 are 100, 200,100, and 200, respectively. Also, assume that there is only one specification, which should be less than or equal to $2.5,1.5,3$, and 2 in the outputs, respectively. The inputs contain 3, 1, and 2 unit(s) of the specification. The costs of buying one unit of the inputs are 6 , 16,10 , respectively, and the price of selling the outputs are $9,15,6$, and 12 .

The P-formulation corresponding to this instance has fourteen variables. Using "Polyopt" package, the chordal extension of the associated graph to the P-formulation (1) has the following maximal cliques:

$$
\begin{aligned}
& \mathcal{D}_{1}=\{3,4,10,13,14\}, \mathcal{D}_{2}=\{1,2,9,11,12\}, \mathcal{D}_{3}=\{1,2,6,9,12\}, \\
& \mathcal{D}_{4}=\{1,5,6,9,12\}, \mathcal{D}_{5}=\{5,6,7,12,13\}, \mathcal{D}_{6}=\{3,7,8,10,13\} \\
& \mathcal{D}_{7}=\{3,8,10,13,14\}
\end{aligned}
$$

As one can see, the overlap in the cliques are a bit high. For example $\{3,8,10,13\}$ is a subset of both $\mathcal{D}_{6}$ and $\mathcal{D}_{7}$. Merging the cliques with the overlaps of larger than 3 results in the following sets of indexes:

$$
\mathcal{D}_{1}=\{3,4,7,8,10,13,14\}, \mathcal{D}_{2}=\{1,2,5,6,9,11,12\}, \mathcal{D}_{3}=\{5,6,7,12,13\}
$$

Now, for the chordal extension of the associated graph to the P-formulation after eliminating 
the equality constraints, the maximal cliques are

$$
\overline{\mathcal{D}}_{1}=\{1,2,3,4,5,6,7,9,10\}, \overline{\mathcal{D}}_{2}=\{3,4,7,8,10\},
$$

where the only difference in them is 8 . Therefore, merging them together results in $\{1, \ldots, 10\}$. So, clearly eliminating the equality constraints destroyed the sparsity pattern of the P-formulation.

\section{B. Additional information about the numerical results}

In the following table, \#var., \#const., size of PSDs, and bold numbers mean the number of linear variables, the number of constraints, the size of semi-definite matrix variable(s), and the optimal value of the instance, respectively. The time in the table contains the time of solving the level of the hierarchy by MOSEK 8.0 [5]. In this table, $n \times m$ in the column "size of PSDs" means that the second level of the sparse-BSOS hierarchy has $n$ semi-definite matrix variables with the size $m \times m$.

Table 5: Some extra information on the level of the sparse-BSOS hierarchy applied to the DTOC problem 11 with the input information $\left(x^{\ell}, x^{u}, x_{0}, x_{N}, u^{\ell}, u^{u}\right)=(0.9,5,1,1,-10,10)$, and $\kappa=2, d=2$. The column "SBSOS without Theorem 55' refers to the result of applying the sparse-BSOS hierarchy applied to the problem after replacing each equality by two inequalities.

\begin{tabular}{|c|c|c|c|c|c|c|c|c|c|c|}
\hline & \multicolumn{9}{|c|}{$\begin{array}{c}\text { SBSOS with } \\
\text { Theorem }\end{array}$} & \multicolumn{4}{c|}{ ThSOS without } \\
$\mathrm{N}$ & value & time & \#var. & \#const. & size of PSDs & value & time & \#var. & \#const. & size of PSDs \\
\hline 50 & $\mathbf{1 . 6 6 0 0}$ & 0.14 & 6,662 & 1,505 & $50 \times 10$ & $\mathbf{1 . 6 6 0 0}$ & 0.19 & 9,452 & 1,505 & $50 \times 10$ \\
\hline 250 & $\mathbf{1 . 6 5 6 9}$ & 0.78 & 33,662 & 7,505 & $250 \times 10$ & $\mathbf{1 . 6 5 6 9}$ & 1.06 & 47,252 & 7,505 & $250 \times 10$ \\
\hline 500 & $\mathbf{1 . 6 5 6 6}$ & 1.65 & 67,412 & 15,005 & $500 \times 10$ & $\mathbf{1 . 6 5 6 6}$ & 2.28 & 94,502 & 15,005 & $500 \times 10$ \\
\hline 1000 & $\mathbf{1 . 6 5 6 4}$ & 3.77 & 134,912 & 30,005 & $1000 \times 10$ & $\mathbf{1 . 6 5 6 4}$ & 4.88 & 189,002 & 30,005 & $1000 \times 10$ \\
\hline 2000 & $\mathbf{1 . 6 5 6 3}$ & 9.14 & 269,912 & 60,005 & $2000 \times 10$ & $\mathbf{1 . 6 5 6 3}$ & 12.68 & 378,002 & 60,005 & $2000 \times 10$ \\
\hline 3000 & $\mathbf{1 . 6 5 6 3}$ & 14.54 & 404,912 & 90,005 & $3000 \times 10$ & $\mathbf{1 . 6 5 6 3}$ & 22.14 & 567,002 & 90,005 & $3000 \times 10$ \\
\hline 4000 & $\approx 1.6563$ & 25.12 & 539,912 & 120,005 & $4000 \times 10$ & $\approx 1.6563$ & 31.14 & 756,002 & 120,005 & $4000 \times 10$ \\
\hline 5000 & $\approx 1.6562$ & 36.33 & 674,912 & 150,005 & $5000 \times 10$ & $\approx 1.6562$ & 54.83 & 945,002 & 150,005 & $5000 \times 10$ \\
\hline
\end{tabular}

